\title{
Effect of Poly(vinyl butyral) Comonomer Sequence on Adhesion to Amorphous Silica: A Coarse-Grained Molecular Dynamics Study
}

\author{
Christopher C. Walker, Jan Genzer, Erik E. Santiso* \\ Department of Chemical and Biomolecular Engineering, North Carolina State University, \\ Raleigh, NC 27695, USA
}

\section{Supporting Information}

*Author to whom correspondence should be addressed. Electronic mail: eesantis@ncsu.edu 


\section{S1. Detailed description of force-mapping procedure}

Forces acting between the coarse-grained PVB copolymer model and all-atom amorphous silica slab model were derived from a series of all-atom MD simulations in which VB and VA monomers were fixed to various $\mathrm{x}, \mathrm{y}$, and $\mathrm{z}$ coordinates relative to the slab over a 3-dimensional (3D) grid. We implemented this in the Large-Scale Atomic/Molecular Massively Parallel Simulator (LAMMPS) software package, ${ }^{1}$ using a harmonic spring with force constant of $500 \mathrm{kcal} / \mathrm{mol} / \AA$ which applies a restoring force to the center of mass of groups of particles at each time step. Each bead of the VB monomer was fixed in separate simulations. The slab center of mass was constrained in a similar manner. The all-atom optimized potentials for liquid simulations (OPLSAA) force field ${ }^{2,3}$ was used to represent both monomers and silica. Periodic boundary conditions were used in the plane of the slab, and fixed boundaries were used in the z-direction (i.e., away from the substrate/polymer interface). Long-range electrostatic interactions for the slab were computed using the particle-particle-particle mesh (PPPM) algorithm. ${ }^{4}$ The time-step was set to $1 \mathrm{fs}$, and a cutoff of $25 \AA$ was used for both Lennard-Jones and electrostatic monomer-silica interactions (i.e., the maximum permissible cutoff for a $50 \AA \times 50 \AA$ cell). For each grid point, forces were averaged over a 250 ps $N V T$ simulation, sampling every $10 \mathrm{fs}$ and discarding the first $50 \mathrm{ps}$ as an equilibration period. In some cases, smaller time steps (i.e., $0.2 \mathrm{fs}$ ) were needed close to the surface due to large repulsive forces. In the attractive region, we used a grid spacing of 2.5 $\AA$ in the $\mathrm{z}$ direction. In the steeper repulsive region, we used a finer spacing of $0.25 \AA$. In the $\mathrm{x}$ and y directions, a grid spacing of $5 \AA$ was used.

To obtain continuous forces and potentials in three dimensions, we first fit the force components as a function of $\mathrm{z}$ coordinate. For each (x,y) line, the repulsive part was fit to a harmonic potential, and the attractive part to a cubic smoothing spline. For each z plane, 2-dimensional (2D) Akima cubic Hermite interpolation, ${ }^{5}$ as implemented in the Matlab ${ }^{6}$ fitting library, was used to complete 
the 3D force field. We chose this interpolant instead of natural splines due to reduced oscillations between grid points. With an analytical form for force as a function of $\mathrm{x}, \mathrm{y}$, and $\mathrm{z}$ coordinates, which decays to zero at $\sim 20 \AA$ from the surface, integration to obtain the corresponding potential is straightforward. We found that the $\mathrm{x}$ and $\mathrm{y}$ components of the monomer-slab force vectors are several orders of magnitude weaker than the $\mathrm{z}$ component for most values of $\mathrm{x}$ and $\mathrm{y}$, and so we only considered the $\mathrm{z}$ component of the force for the adhesion simulations. To implement the force mapping in LAMMPS, we developed a $\mathrm{C}++$ code in the context of the 'fix external' framework, which at each time step extracts atom type and coordinates, performs trilinear interpolation on tabulated bead-slab force and potential values, and adds the forces to each bead. 


\section{S2. Contact maps of each of the 5 PVB coarse-grained bead types with the force-mapped silica surface.}
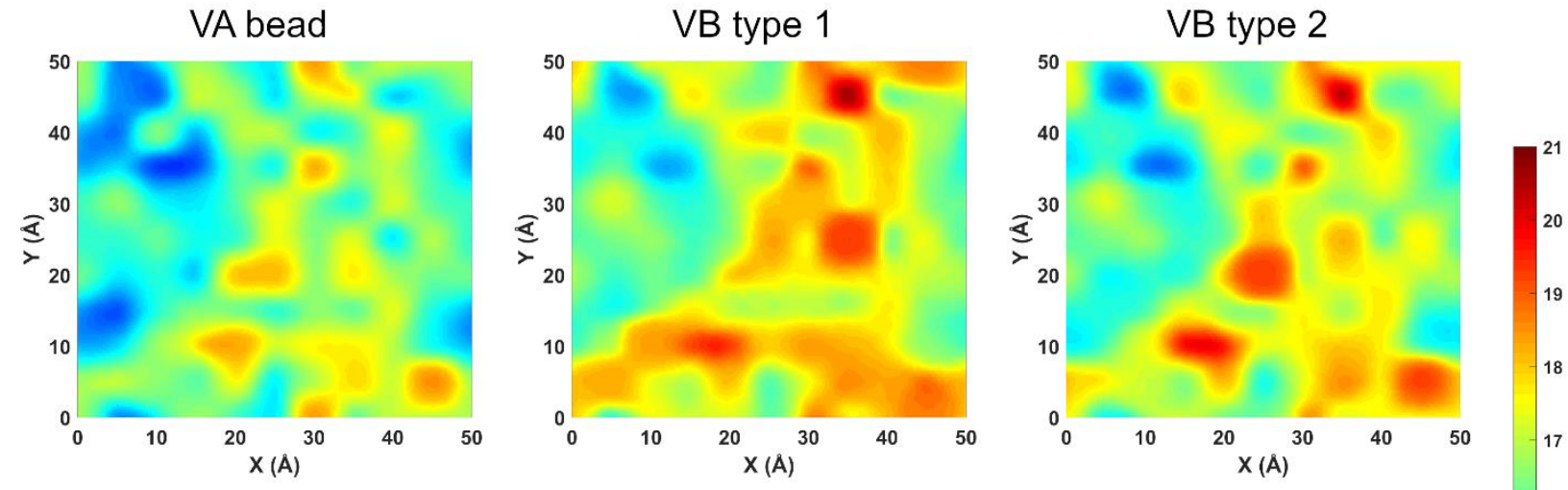

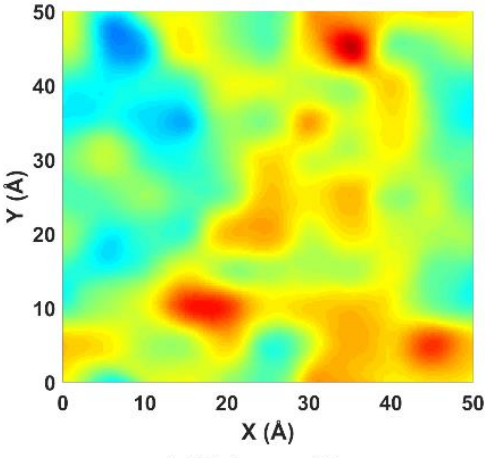

VB type 3

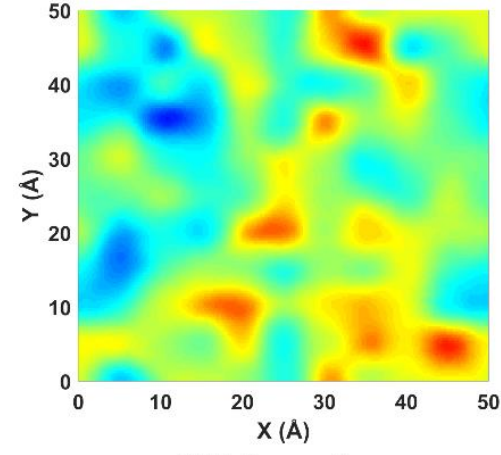

VB type 4

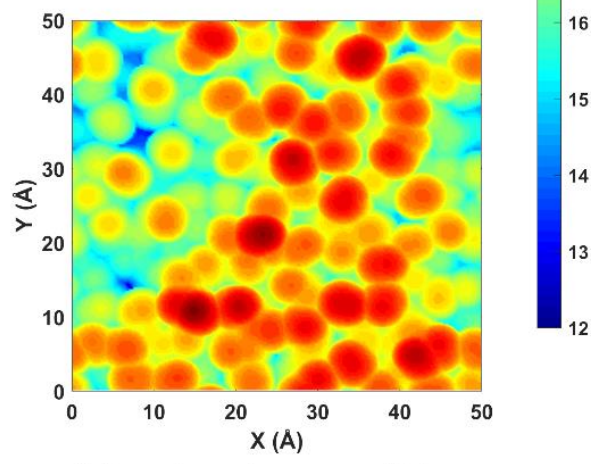

AA solvent-accessible surface

Figure S1. Contact maps of each of the 5 PVB coarse-grained bead types with the force-mapped silica surface. VB bead types 1,2,3 and 4 correspond to the chemical fragments based on 2,5dimethyltetrahydrofuran, paraldehyde, 1,4-dioxane, and $n$-butane, respectively. In the lower right, a hard contact map of the solvent-accessible all-atom surface is shown for reference, computed using a spherical probe of radius $1.75 \AA$. Colors represent lowest points in the z-direction (i.e., normal to the PVB-silica interface) sampled by a PVB bead, in Angstroms. For the coarse-grained contact maps, these were obtained from MD simulations of adsorbed PVB or PVA homopolymer melts at $400 \mathrm{~K}$. The base of the silica slab model is located near $\mathrm{z}=0$. The coarse-grained bead contact maps are smoothed using the user-created Matlab smoothn function available through the Matlab File Exchange. 
S3. Additional adhesion simulation results: reproducibility of contact fraction over 3 simulations with independent sequences (100 ns average). Dark red represents areas of exclusive VA contacts, and dark blue represents areas of exclusive VB contacts. Coordinates are given in Angstroms.

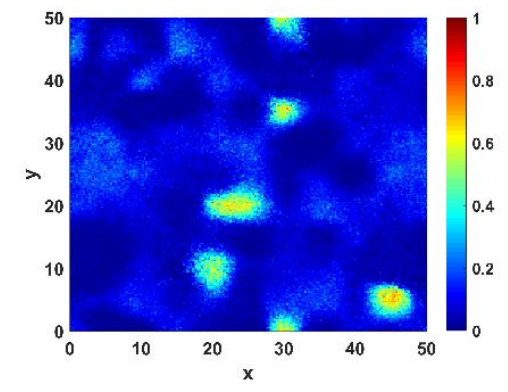

$$
\mathrm{b}=0.00,25 \mathrm{~mol} \% \mathrm{VA}
$$
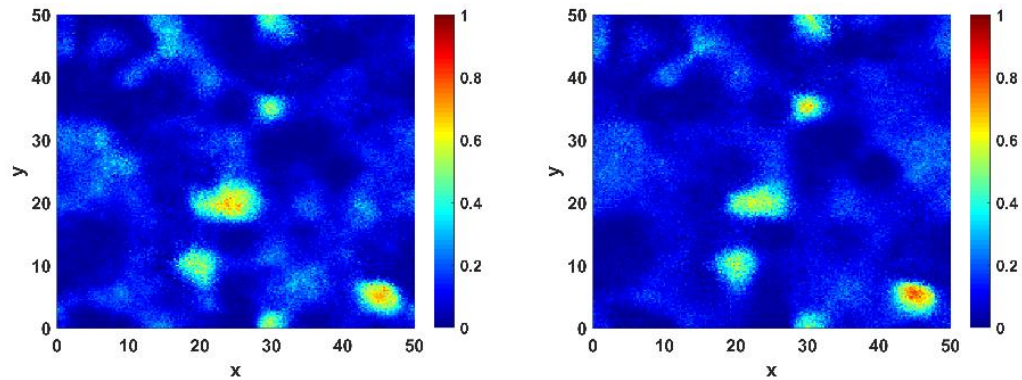

Figure S2. Contact fraction histograms of VA monomers on the force-mapped silica surface as a function of $\mathrm{x}$ and $\mathrm{y}$, for three independent sequence distributions with blockiness $\mathrm{b}=0.00$ and 25 mol\% VA content.

$$
\mathrm{b}=0.25,25 \mathrm{~mol} \% \mathrm{VA}
$$
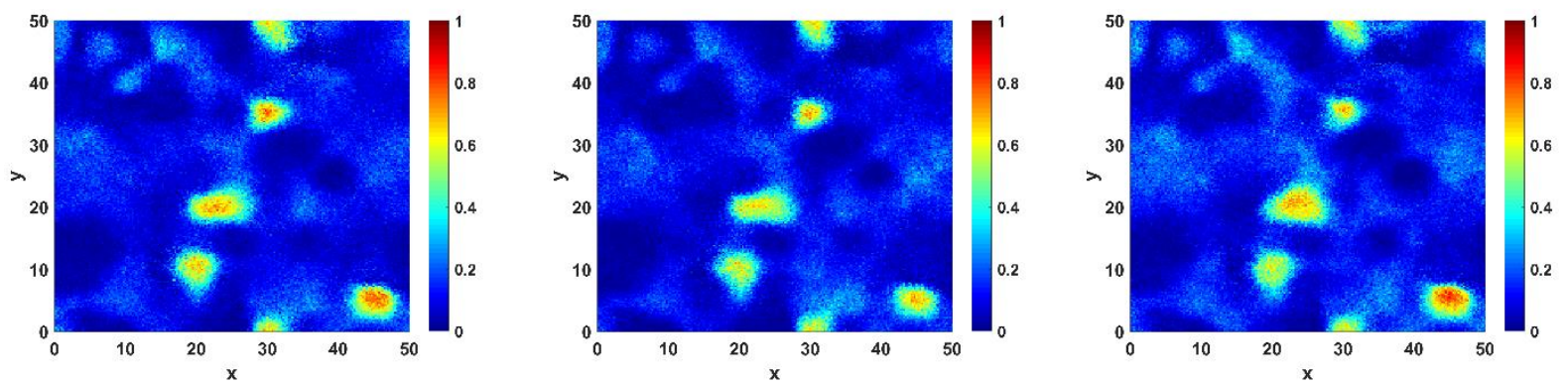

Figure S3. Contact fraction histograms of VA monomers on the force-mapped silica surface as a function of $\mathrm{x}$ and $\mathrm{y}$, for three independent sequence distributions with blockiness $b=0.25$ and 25 mol\% VA content.
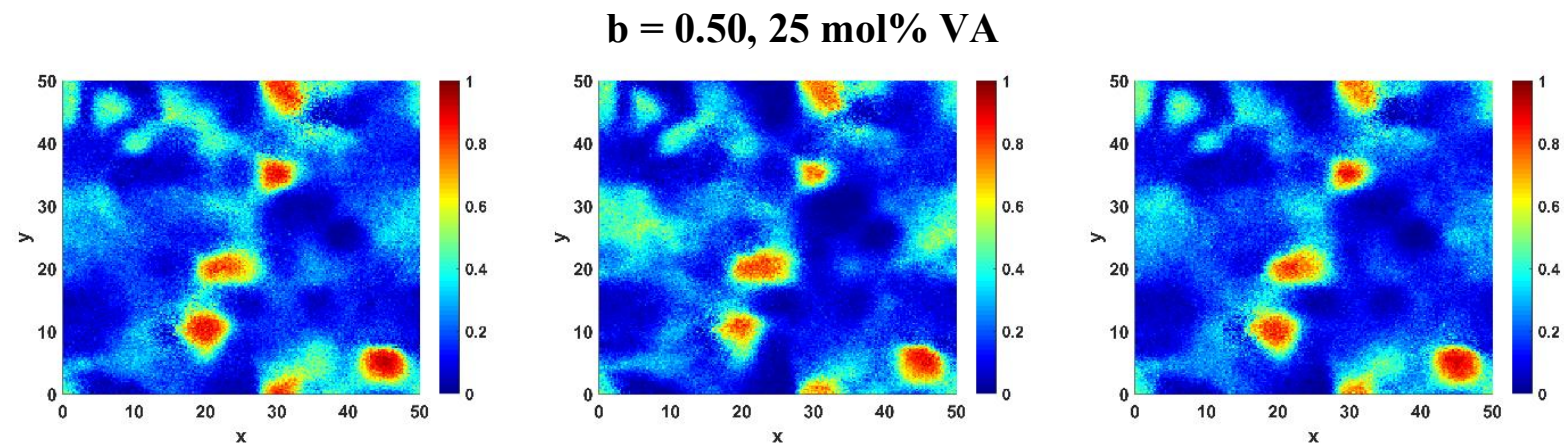

Figure S4. Contact fraction histograms of VA monomers on the force-mapped silica surface as a function of $x$ and $y$, for three independent sequence distributions with blockiness $b=0.50$ and 25 mol\% VA content. 

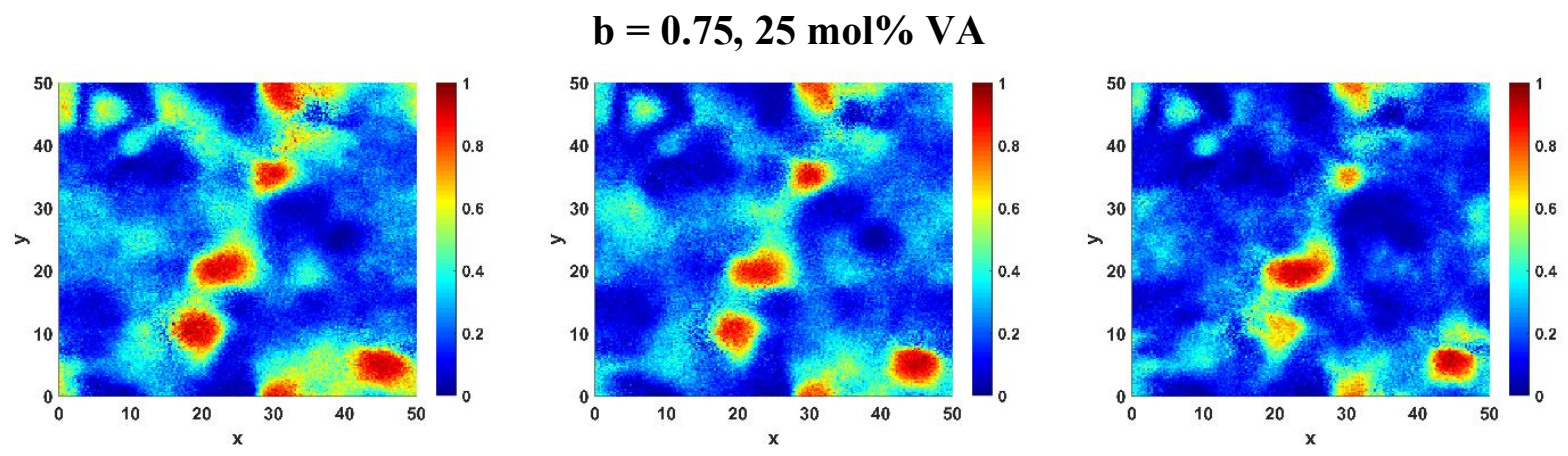

Figure S5. Contact fraction histograms of VA monomers on the force-mapped silica surface as a function of $x$ and $y$, for three independent sequence distributions with blockiness $b=0.75$ and 25 mol\% VA content.
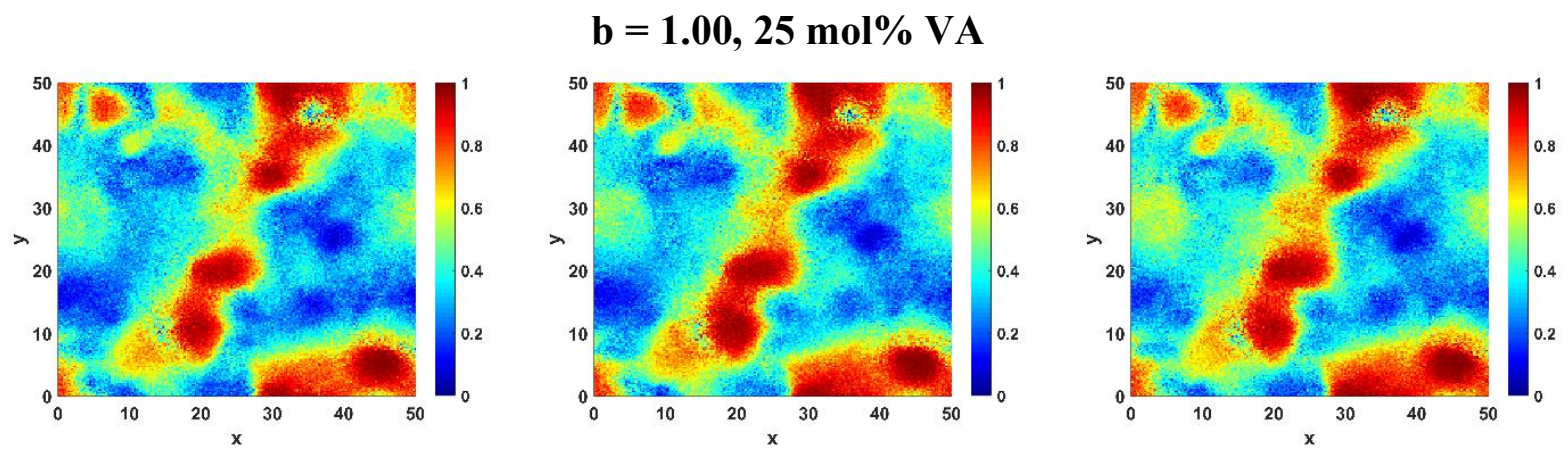

Figure S6. Contact fraction histograms of VA monomers on the force-mapped silica surface as a function of $\mathrm{x}$ and $\mathrm{y}$, for three independent sequence distributions with blockiness $b=1.00$ and 25 mol\% VA content.
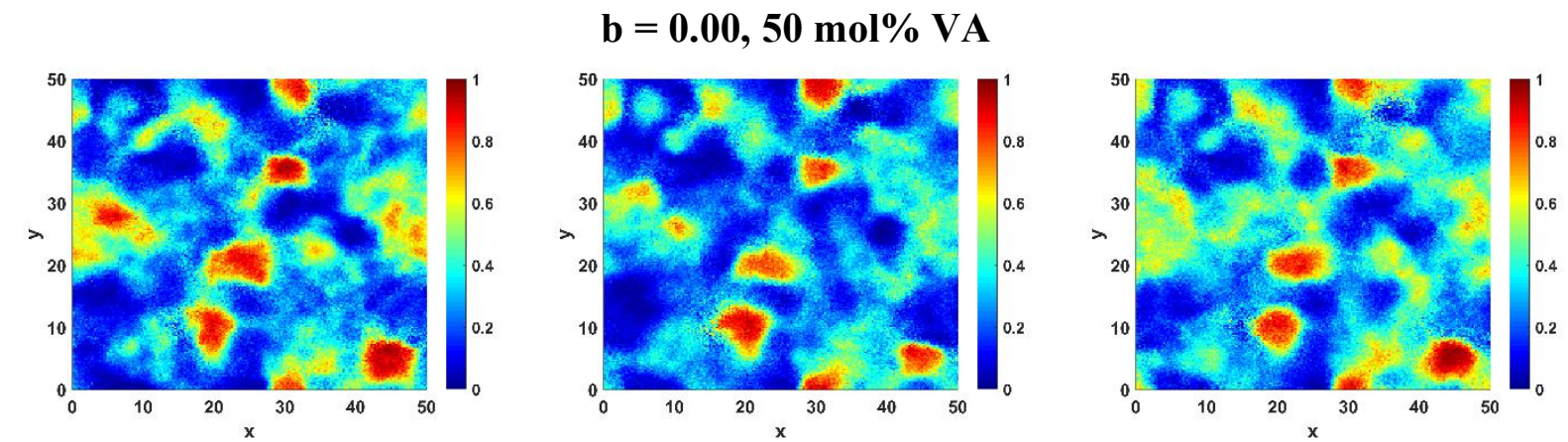

Figure S7. Contact fraction histograms of VA monomers on the force-mapped silica surface as a function of $\mathrm{x}$ and $\mathrm{y}$, for three independent sequence distributions with blockiness $\mathrm{b}=0.00$ and 50 mol\% VA content. 

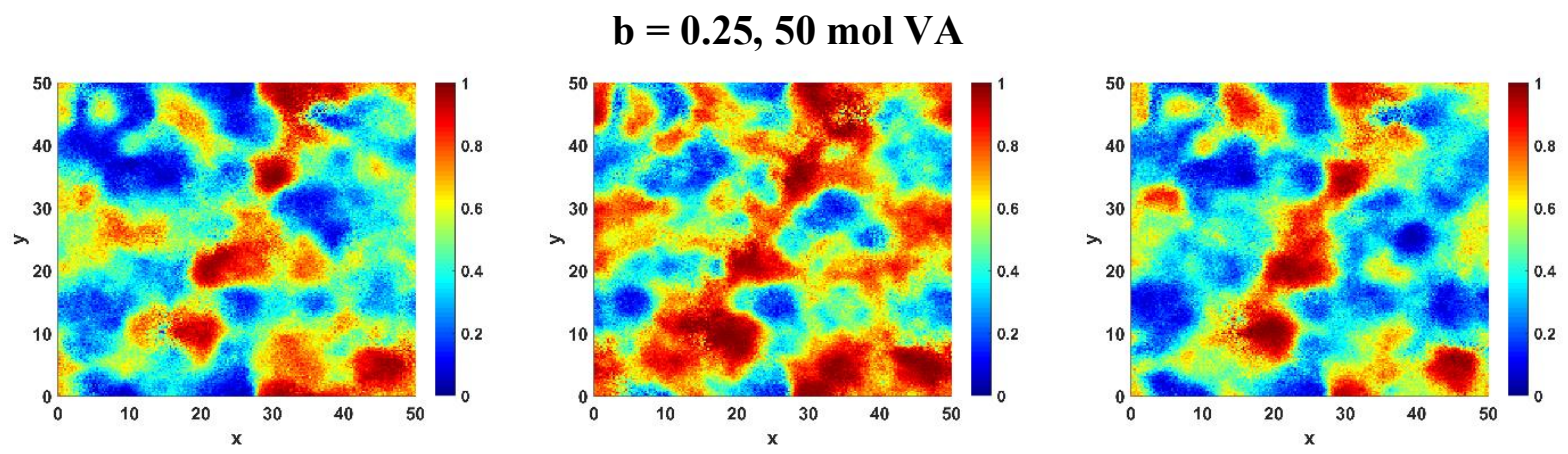

Figure S8. Contact fraction histograms of VA monomers on the force-mapped silica surface as a function of $\mathrm{x}$ and $\mathrm{y}$, for three independent sequence distributions with blockiness $b=0.25$ and 50 mol\% VA content.
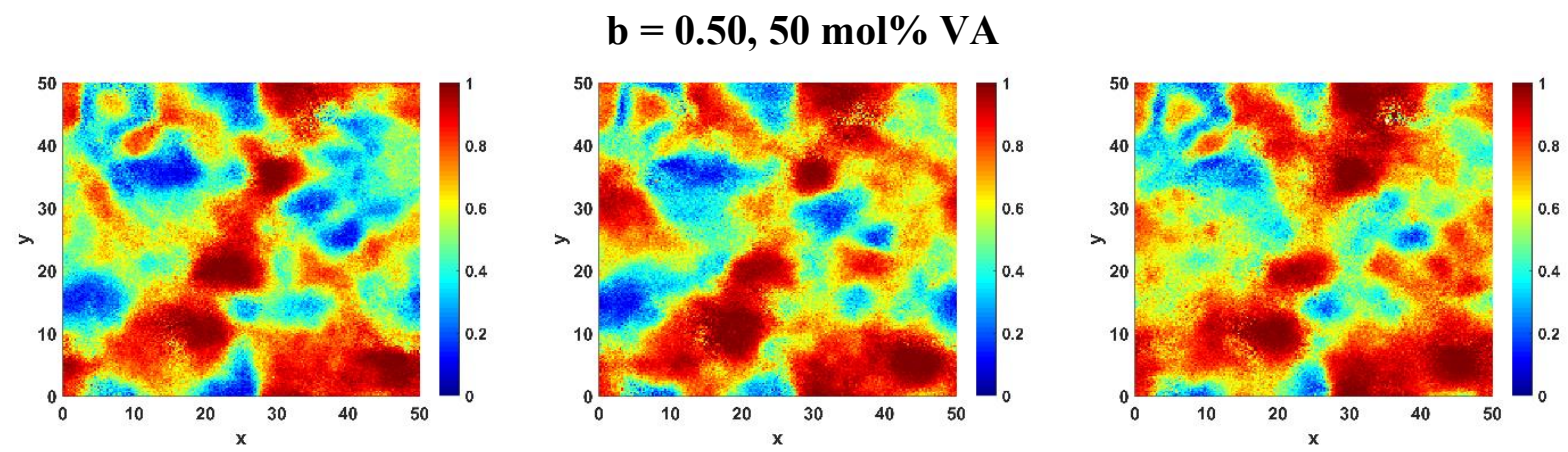

Figure S9. Contact fraction histograms of VA monomers on the force-mapped silica surface as a function of $\mathrm{x}$ and $\mathrm{y}$, for three independent sequence distributions with blockiness $\mathrm{b}=0.50$ and 50 mol\% VA content.

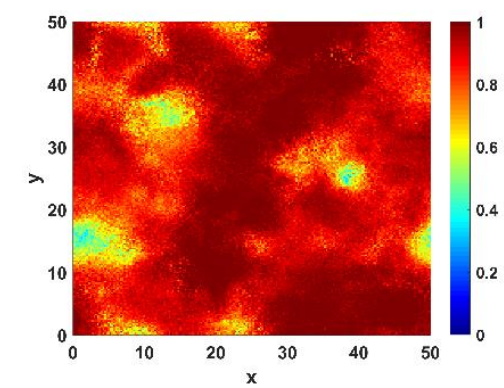

$$
\mathrm{b}=\mathbf{0 . 7 5}, 50 \mathrm{~mol} \% \mathrm{VA}
$$

Figure S10. Contact fraction histograms of VA monomers on the force-mapped silica surface as a function of $x$ and $y$, for three independent sequence distributions with blockiness $b=0.75$ and $50 \mathrm{~mol} \%$ VA content. 


$$
\mathrm{b}=1.00,50 \mathrm{~mol} \% \mathrm{VA}
$$
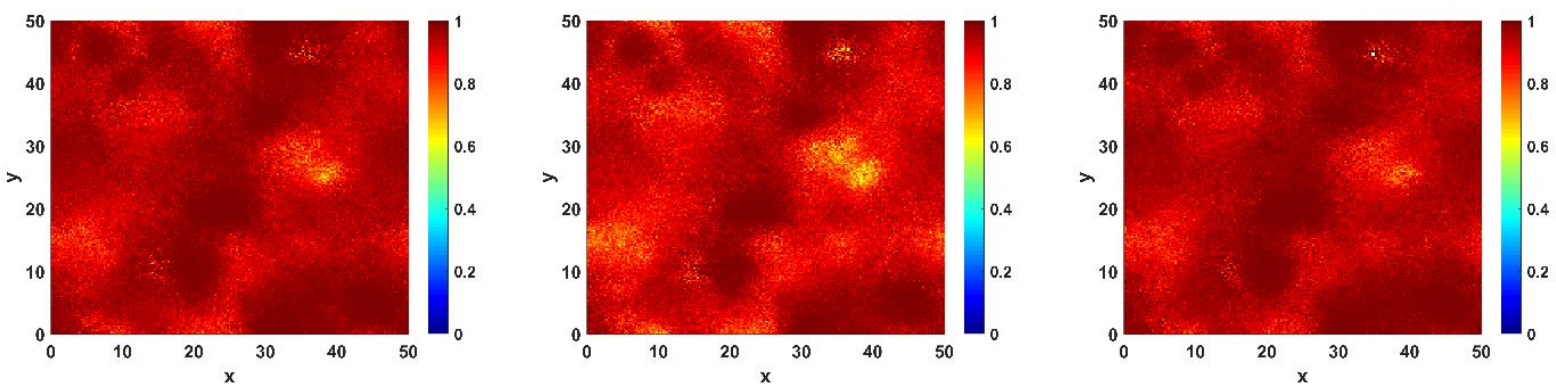

Figure S11. Contact fraction histograms of VA monomers on the force-mapped silica surface as a function of $\mathrm{x}$ and $\mathrm{y}$, for three independent sequence distributions with blockiness $\mathrm{b}=1.00$ and $50 \mathrm{~mol} \%$ VA content.
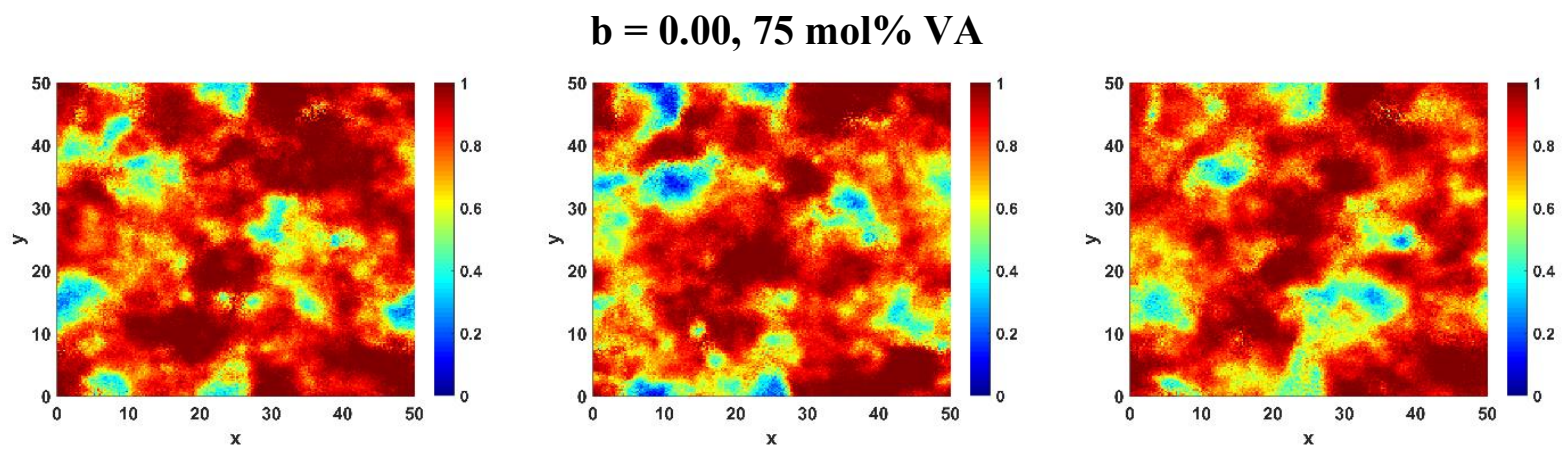

Figure S12. Contact fraction histograms of VA monomers on the force-mapped silica surface as a function of $\mathrm{x}$ and $\mathrm{y}$, for three independent sequence distributions with blockiness $\mathrm{b}=0.00$ and $75 \mathrm{~mol} \%$ VA content.

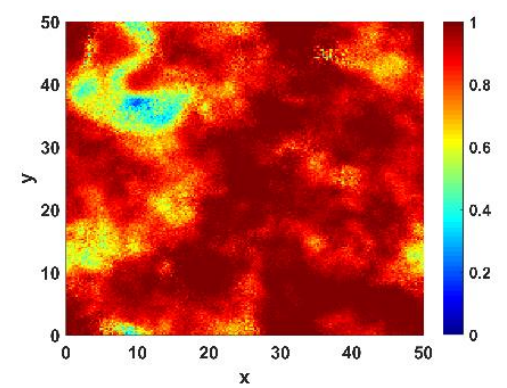

$$
\mathbf{b}=0.25,75 \mathrm{~mol} \% \mathrm{VA}
$$
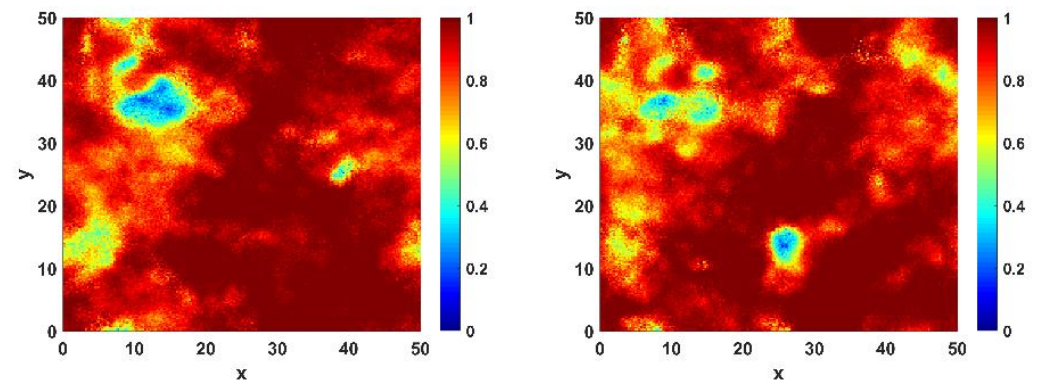

Figure S13. Contact fraction histograms of VA monomers on the force-mapped silica surface as a function of $\mathrm{x}$ and $\mathrm{y}$, for three independent sequence distributions with blockiness $\mathrm{b}=0.25$ and $75 \mathrm{~mol} \%$ VA content. 


$$
\mathrm{b}=0.50,75 \mathrm{~mol} \% \mathrm{VA}
$$
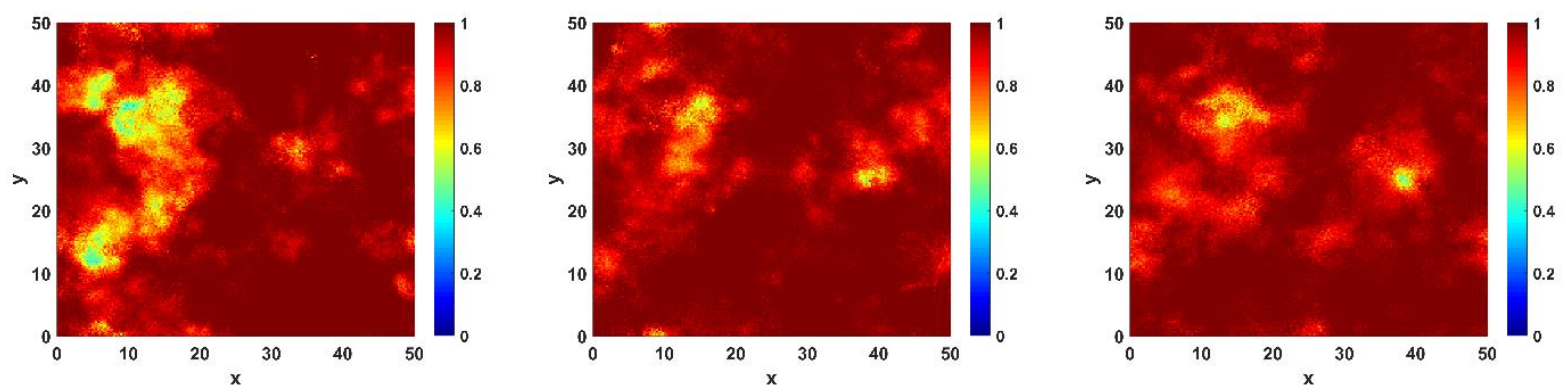

Figure S14. Contact fraction histograms of VA monomers on the force-mapped silica surface as a function of $\mathrm{x}$ and $\mathrm{y}$, for three independent sequence distributions with blockiness $\mathrm{b}=0.50$ and $75 \mathrm{~mol} \%$ VA content.

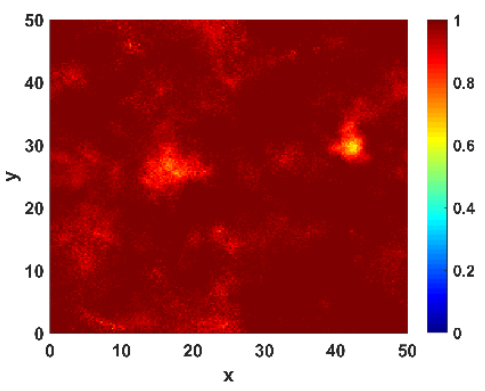

$$
\text { b }=0.75,75 \mathrm{~mol} \% \mathrm{VA}
$$
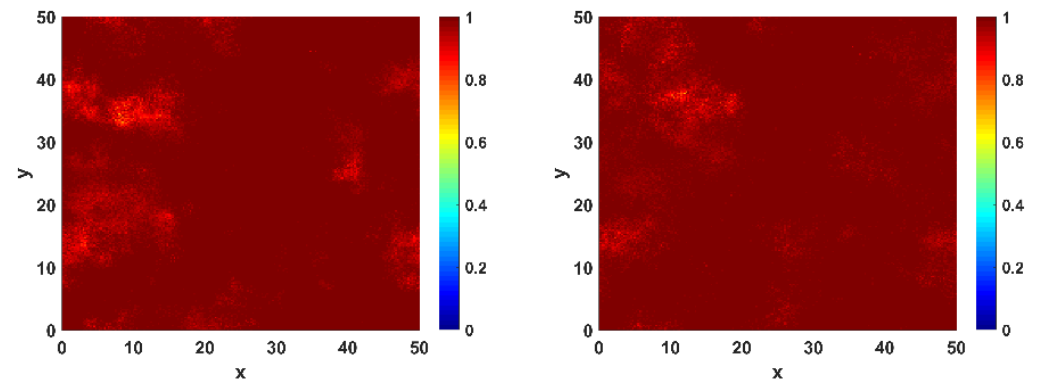

Figure S15. Contact fraction histograms of VA monomers on the force-mapped silica surface as a function of $\mathrm{x}$ and $\mathrm{y}$, for three independent sequence distributions with blockiness $\mathrm{b}=0.75$ and $75 \mathrm{~mol} \%$ VA content.

$$
\text { b }=1.00,75 \mathrm{~mol} \% \mathrm{VA}
$$
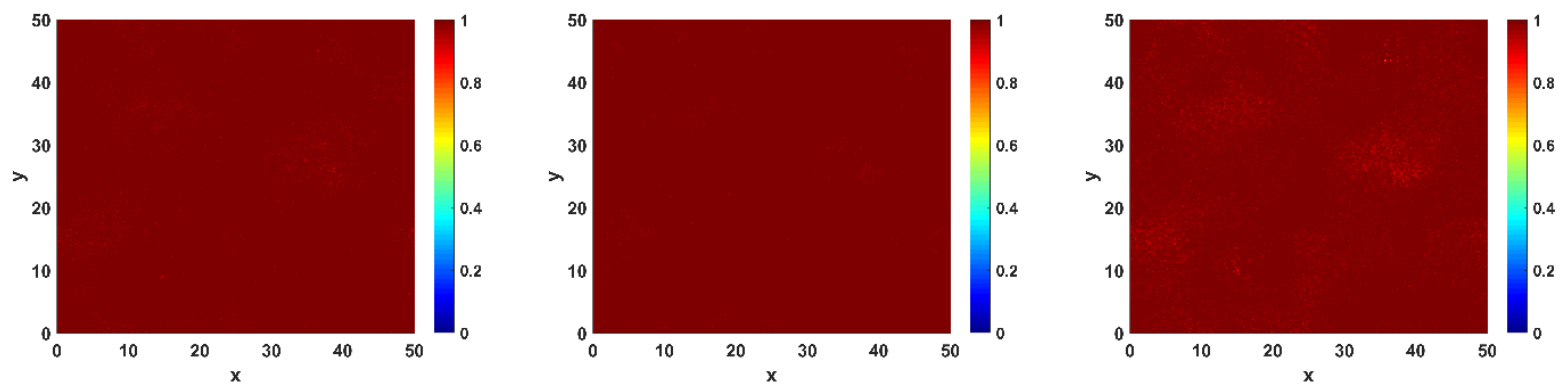

Figure S16. Contact fraction histograms of VA monomers on the force-mapped silica surface as a function of $\mathrm{x}$ and $\mathrm{y}$, for three independent sequence distributions with blockiness $\mathrm{b}=1.00$ and $75 \mathrm{~mol} \%$ VA content. 
S4. Additional adhesion simulation results: morphology volume renderings for all three independent trials at 150ns snapshot $($ red $=V A$, cyan $=V B)$. Beads are represented by spheres defined by their Mie diameter, scaled by their shape factor. All coordinates are given in Angstroms.
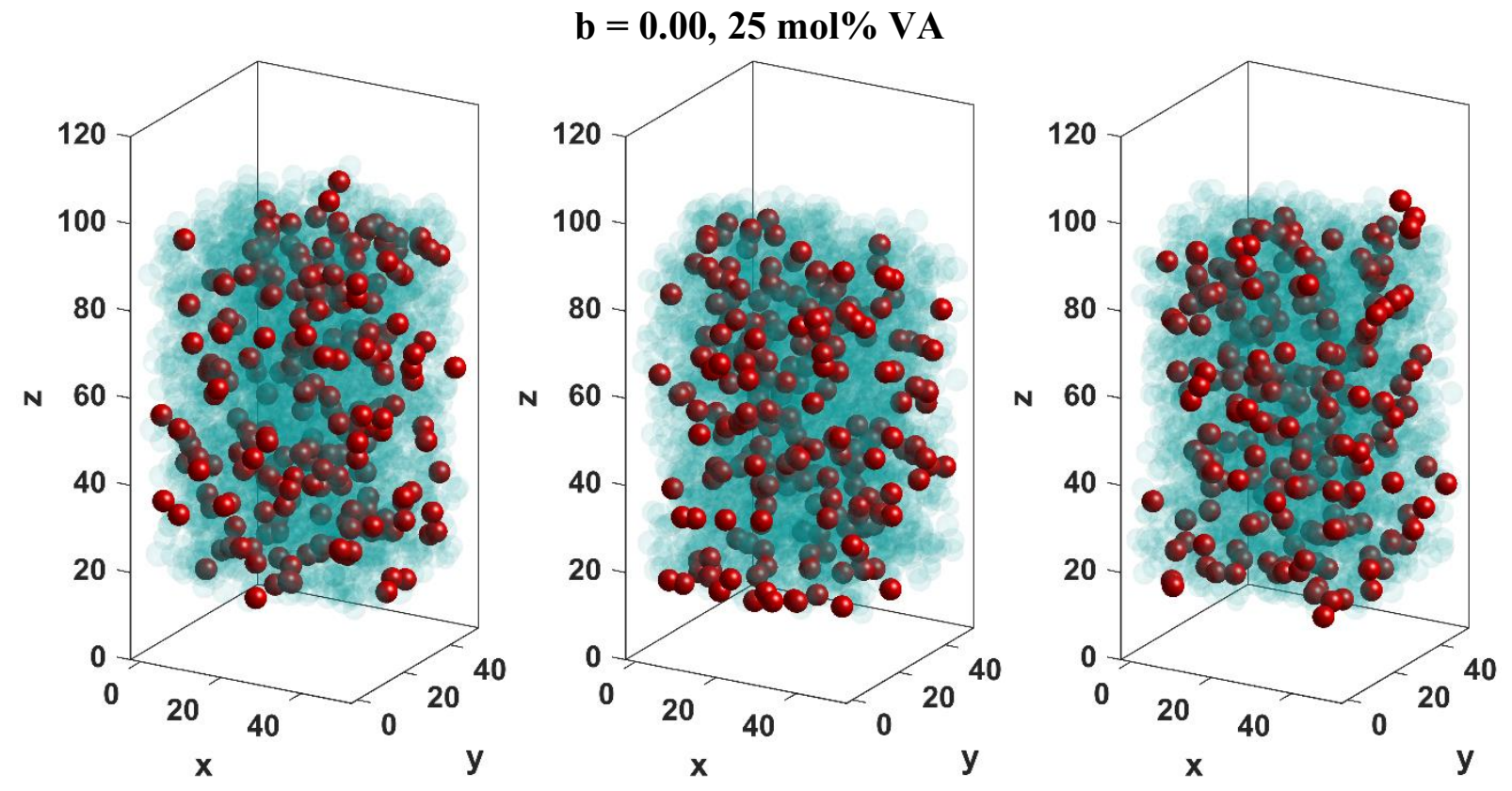

Figure S17. Morphology rendering for three independent sequence distributions with blockiness $\mathrm{b}=0.00$ and $25 \mathrm{~mol} \%$ VA content.
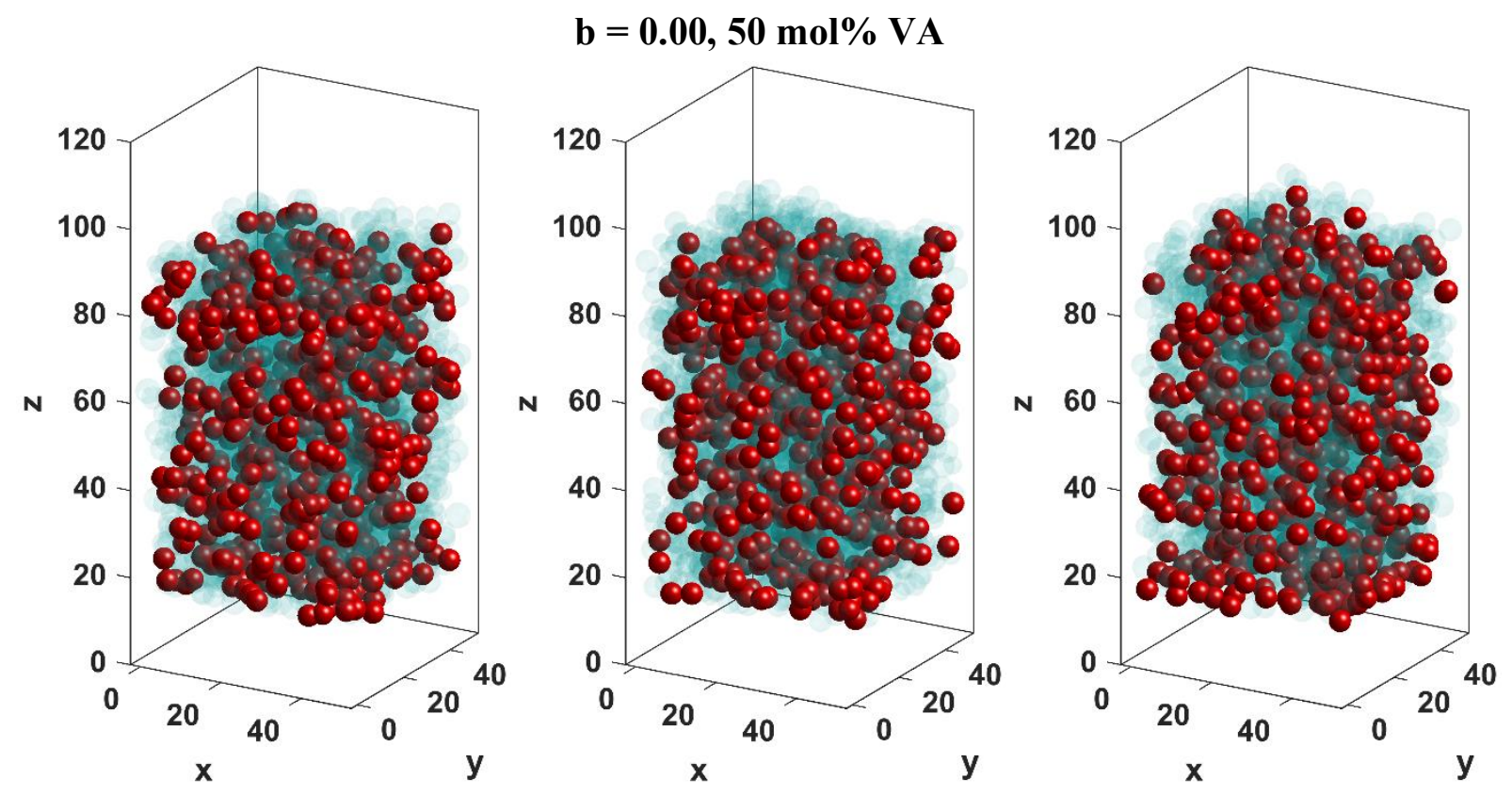

Figure S18. Morphology rendering for three independent sequence distributions with blockiness $\mathrm{b}=0.00$ and $50 \mathrm{~mol} \%$ VA content. 

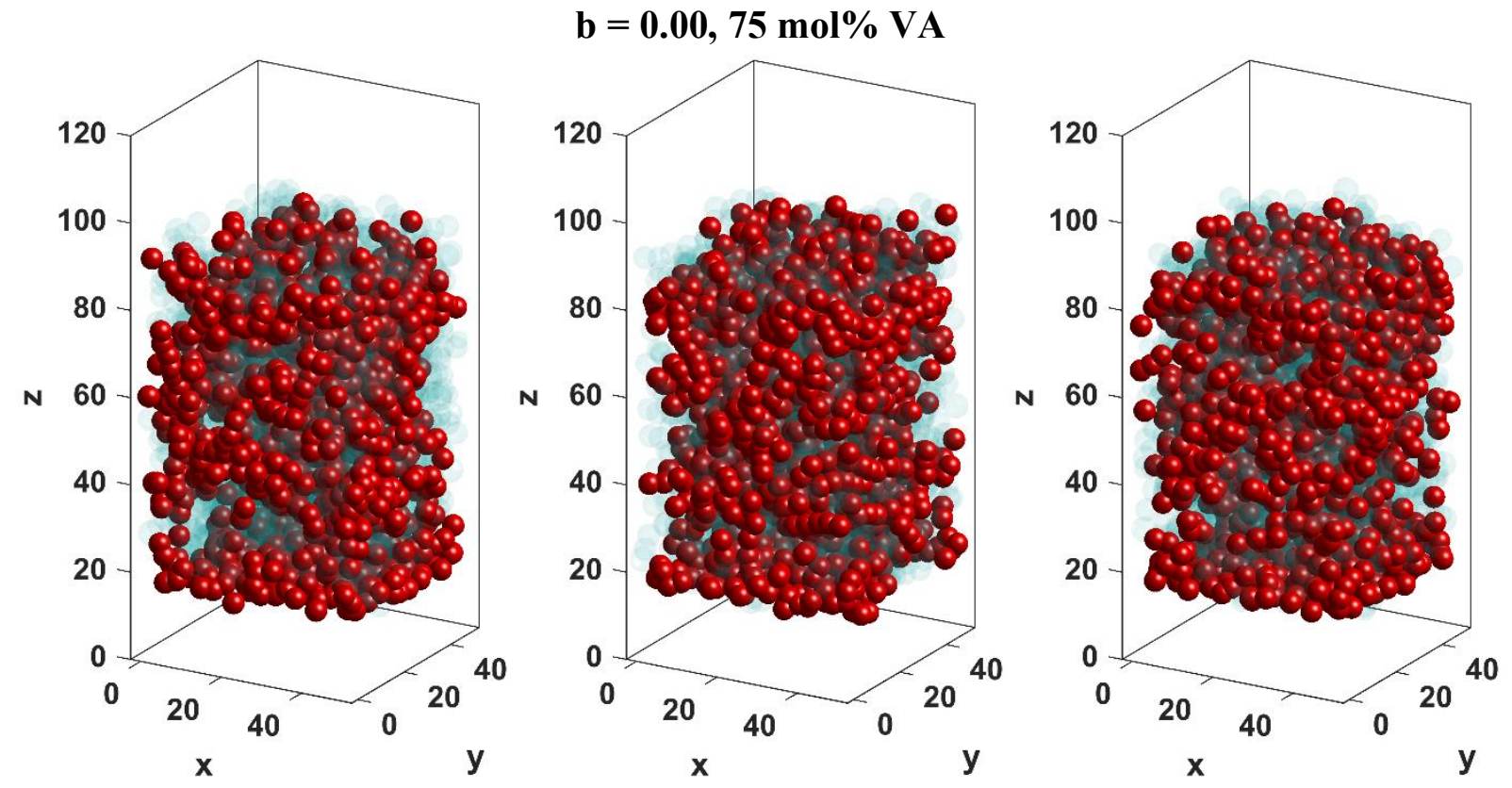

Figure S19. Morphology rendering for three independent sequence distributions with blockiness $\mathrm{b}=0.00$ and $75 \mathrm{~mol} \% \mathrm{VA}$ content.
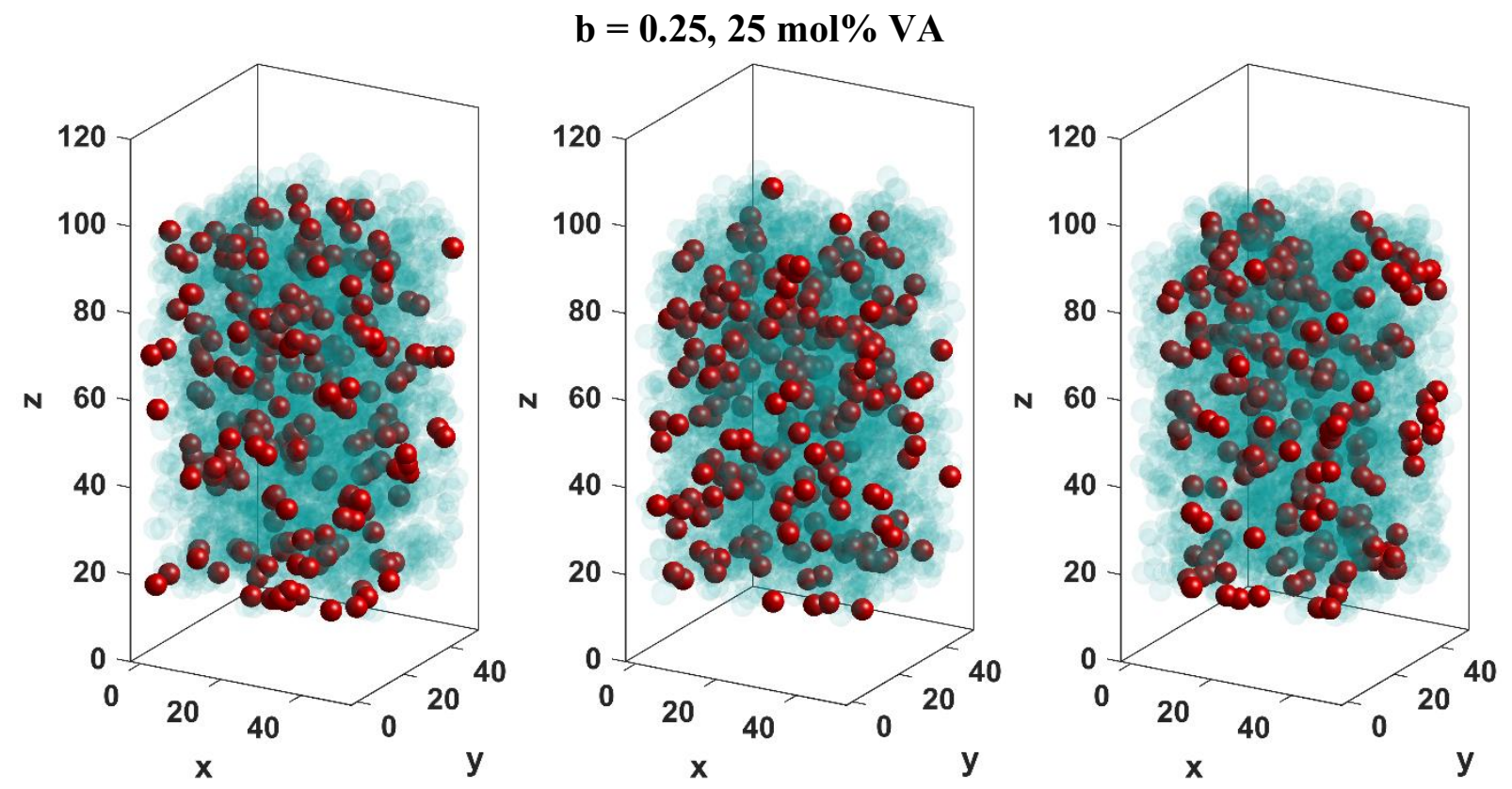

Figure S20. Morphology rendering for three independent sequence distributions with blockiness $\mathrm{b}=0.25$ and $25 \mathrm{~mol} \%$ VA content. 

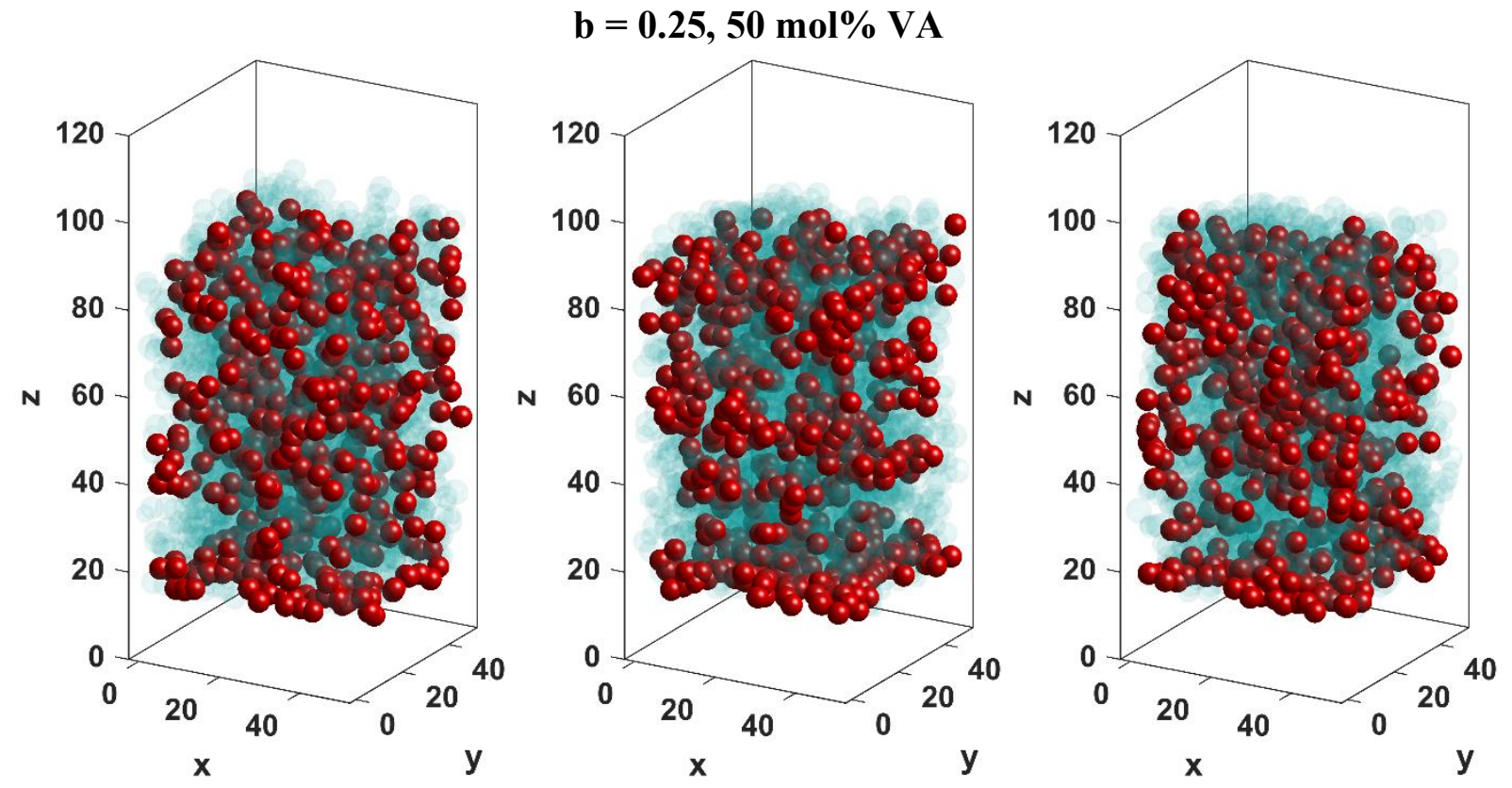

Figure S21. Morphology rendering for three independent sequence distributions with blockiness $\mathrm{b}=0.25$ and $50 \mathrm{~mol} \%$ VA content.
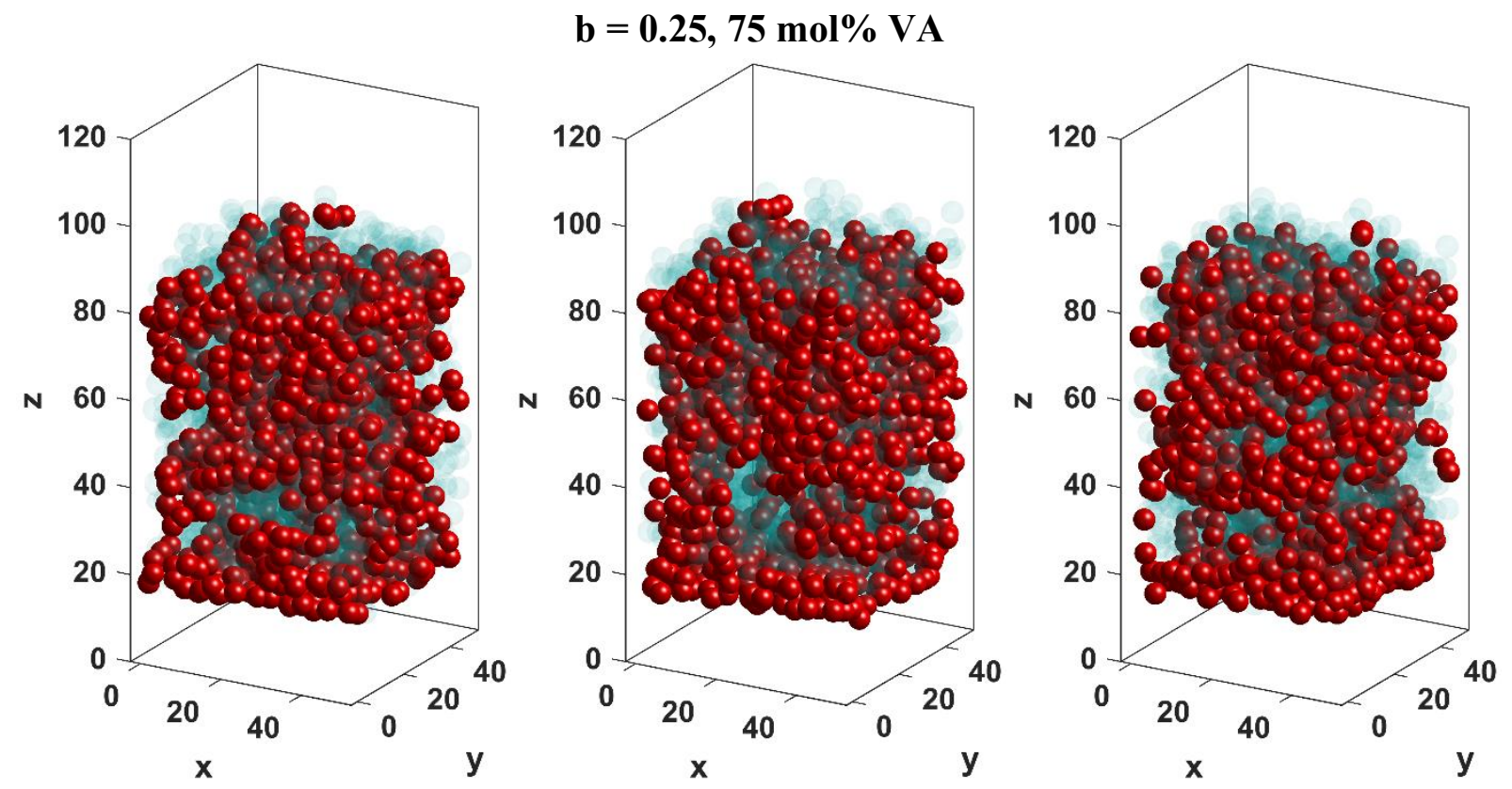

Figure S22. Morphology rendering for three independent sequence distributions with blockiness $\mathrm{b}=0.25$ and $75 \mathrm{~mol} \%$ VA content. 

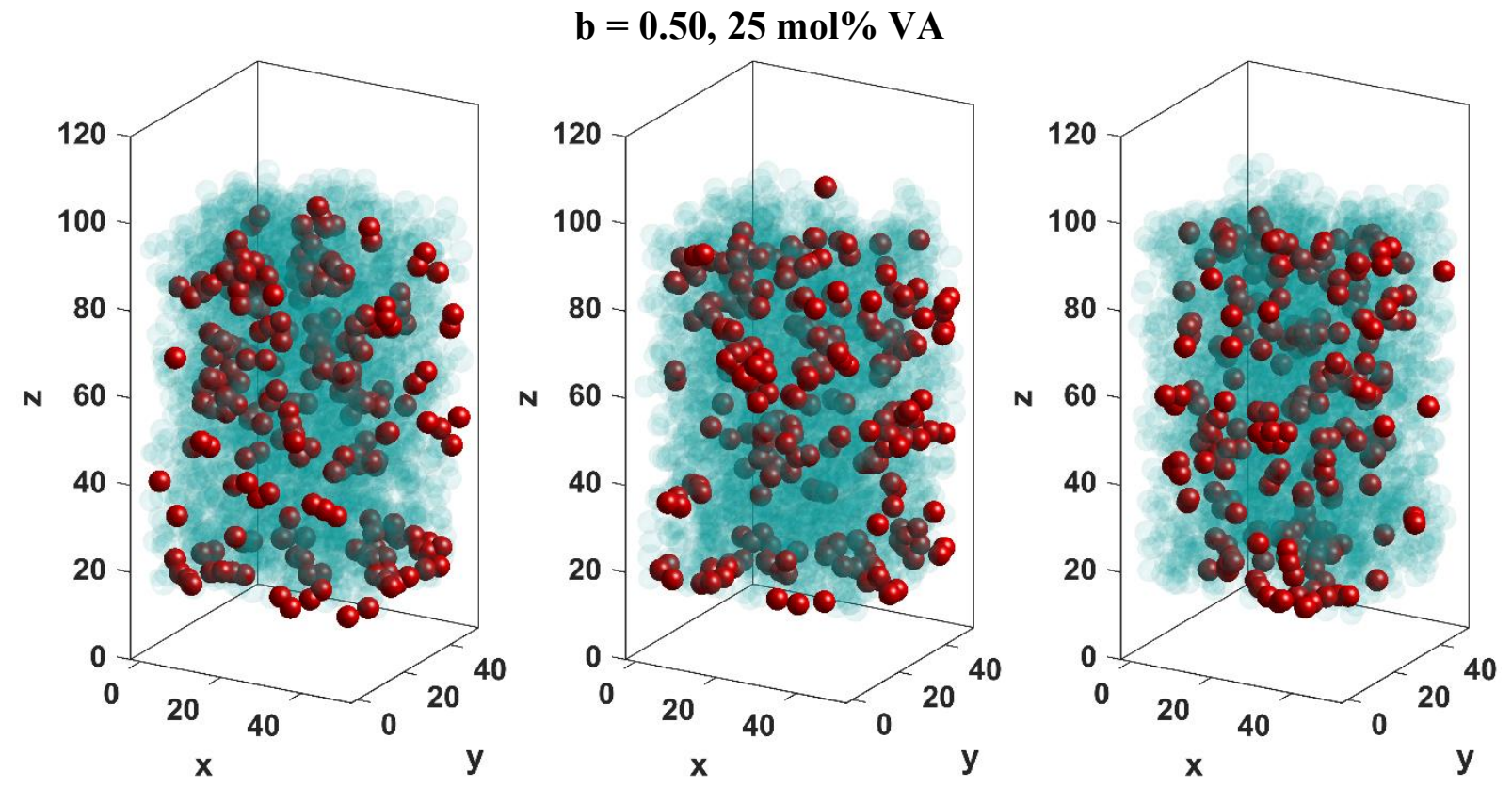

Figure S23. Morphology rendering for three independent sequence distributions with blockiness $\mathrm{b}=0.50$ and $25 \mathrm{~mol} \% \mathrm{VA}$ content.
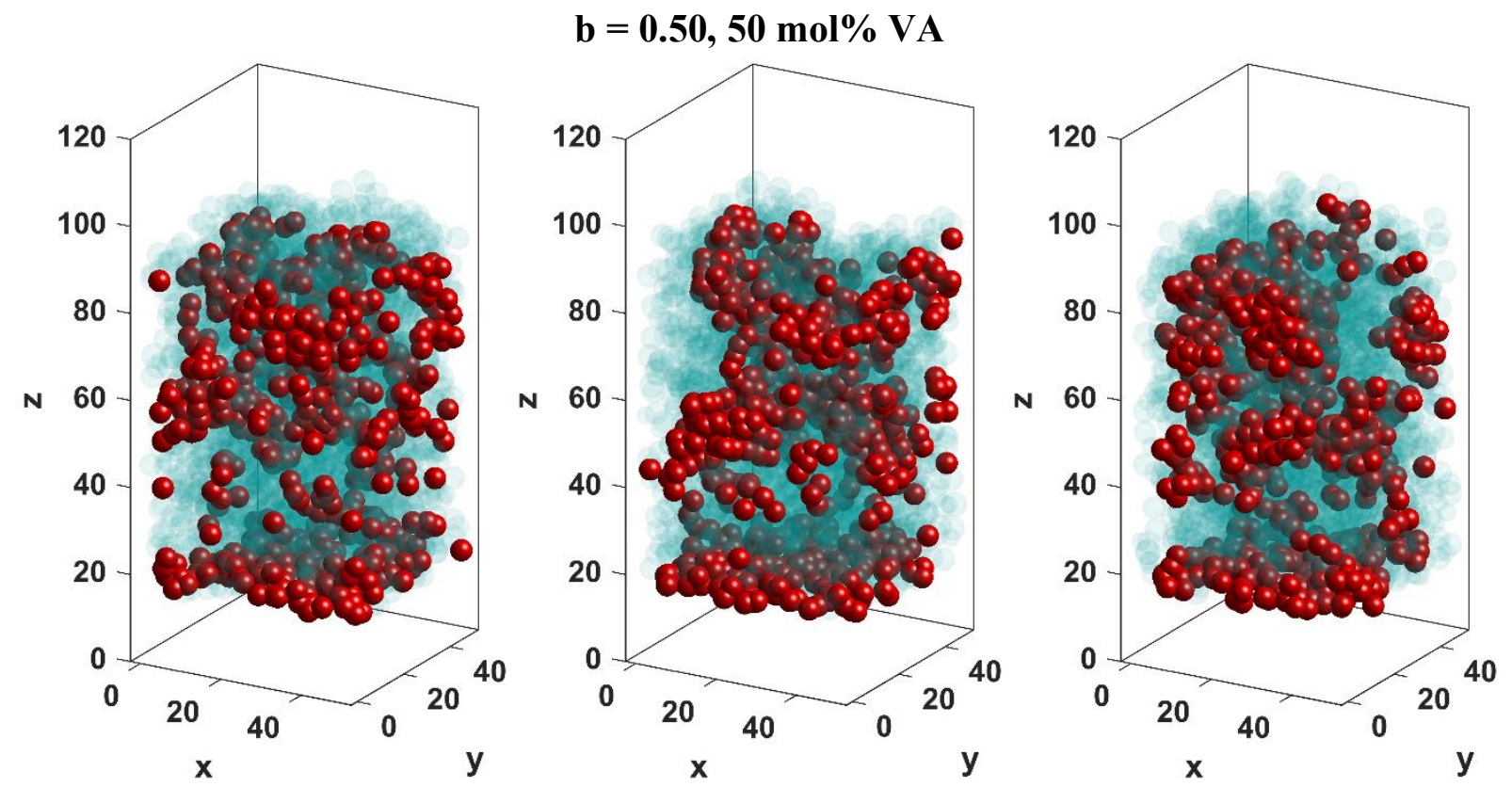

Figure S24. Morphology rendering for three independent sequence distributions with blockiness $\mathrm{b}=0.50$ and $50 \mathrm{~mol} \%$ VA content. 

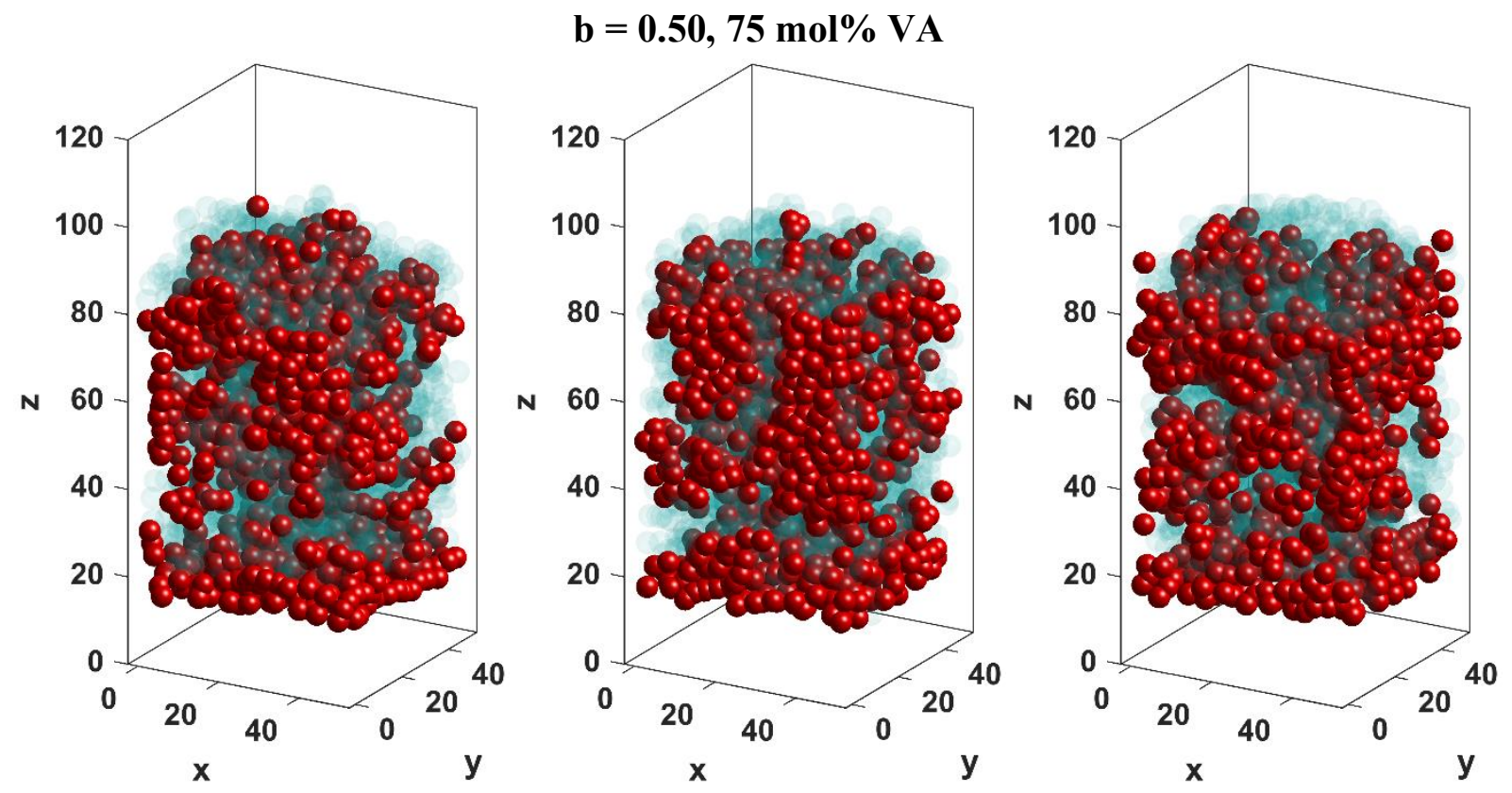

Figure S25. Morphology rendering for three independent sequence distributions with blockiness $\mathrm{b}=0.50$ and $75 \mathrm{~mol} \% \mathrm{VA}$ content.
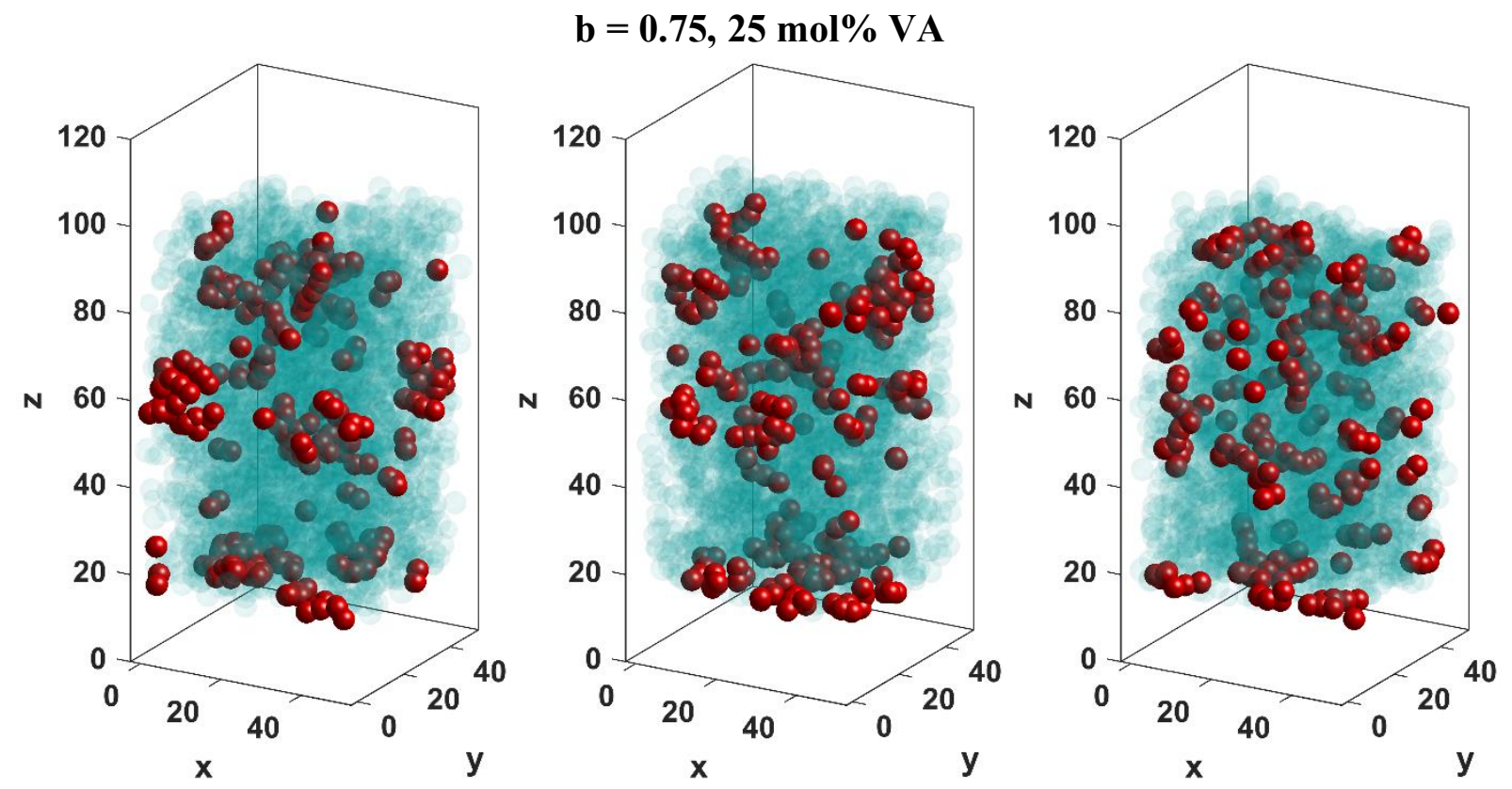

Figure S26. Morphology rendering for three independent sequence distributions with blockiness $\mathrm{b}=0.75$ and $25 \mathrm{~mol} \%$ VA content. 

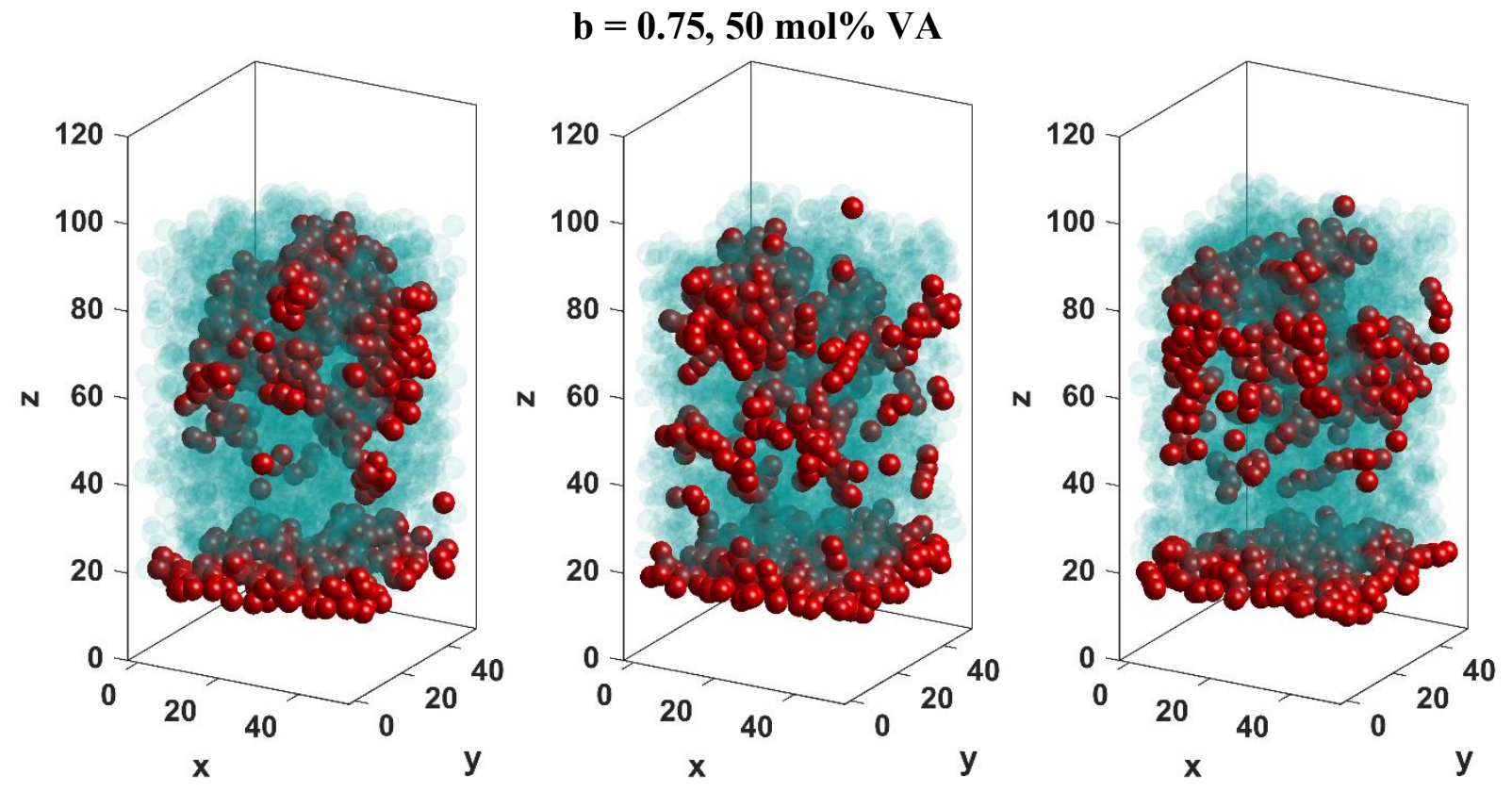

Figure S27. Morphology rendering for three independent sequence distributions with blockiness $\mathrm{b}=0.75$ and $50 \mathrm{~mol} \% \mathrm{VA}$ content.
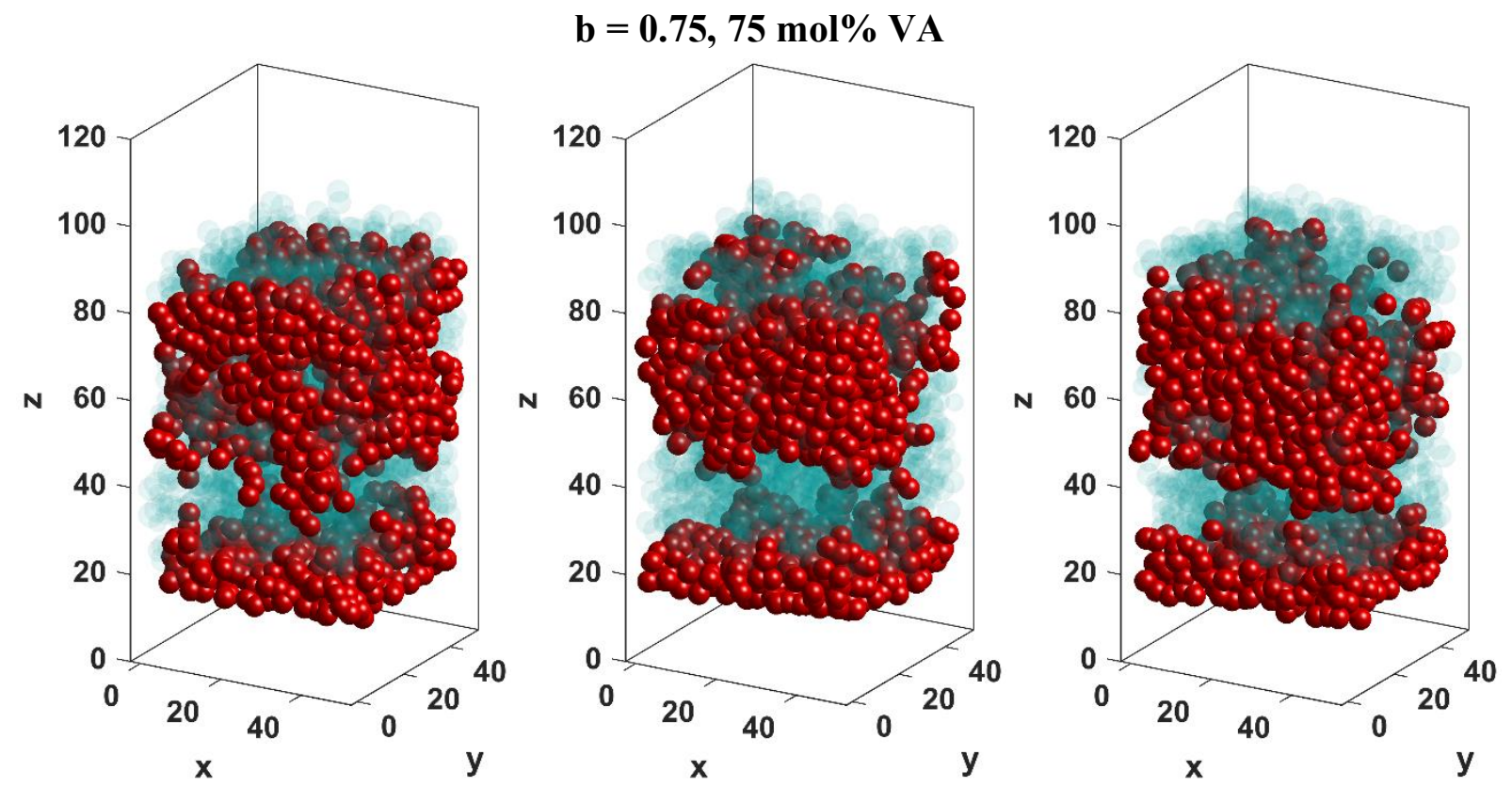

Figure S28. Morphology rendering for three independent sequence distributions with blockiness $\mathrm{b}=0.75$ and $75 \mathrm{~mol} \%$ VA content. 

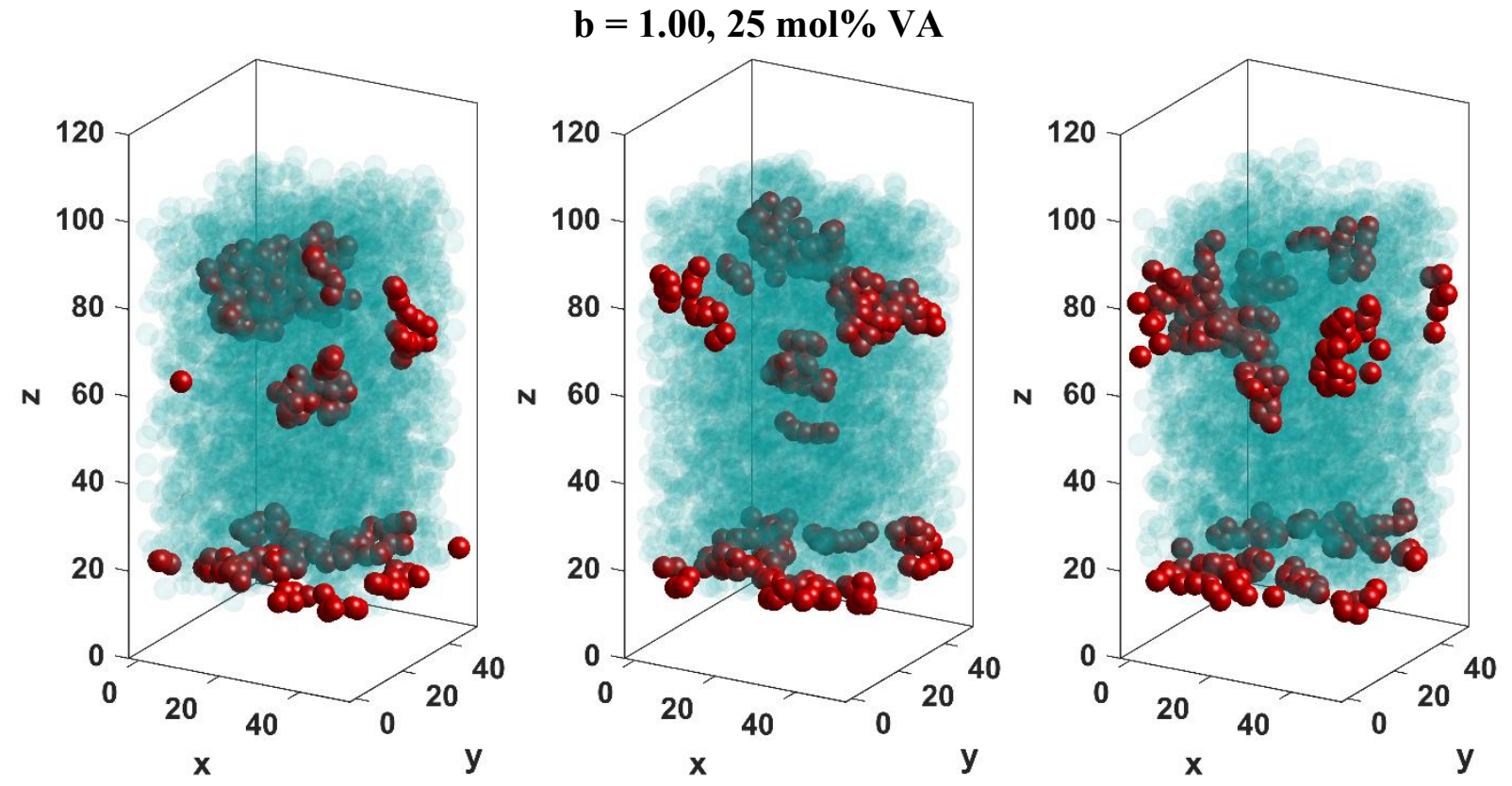

Figure S29. Morphology rendering for three independent sequence distributions with blockiness $\mathrm{b}=1.00$ and $25 \mathrm{~mol} \% \mathrm{VA}$ content.
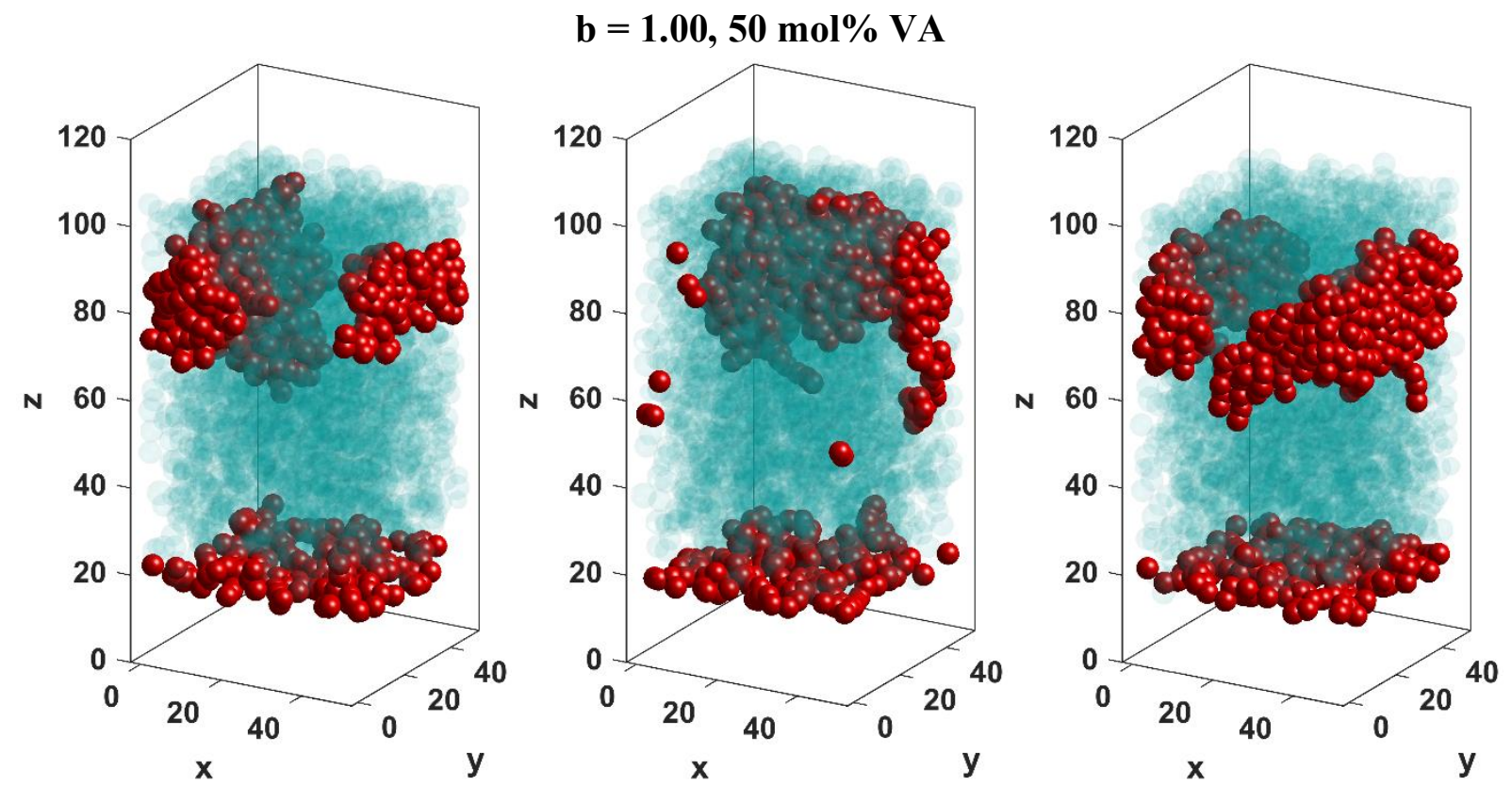

Figure S30. Morphology rendering for three independent sequence distributions with blockiness $\mathrm{b}=1.00$ and $50 \mathrm{~mol} \%$ VA content. 

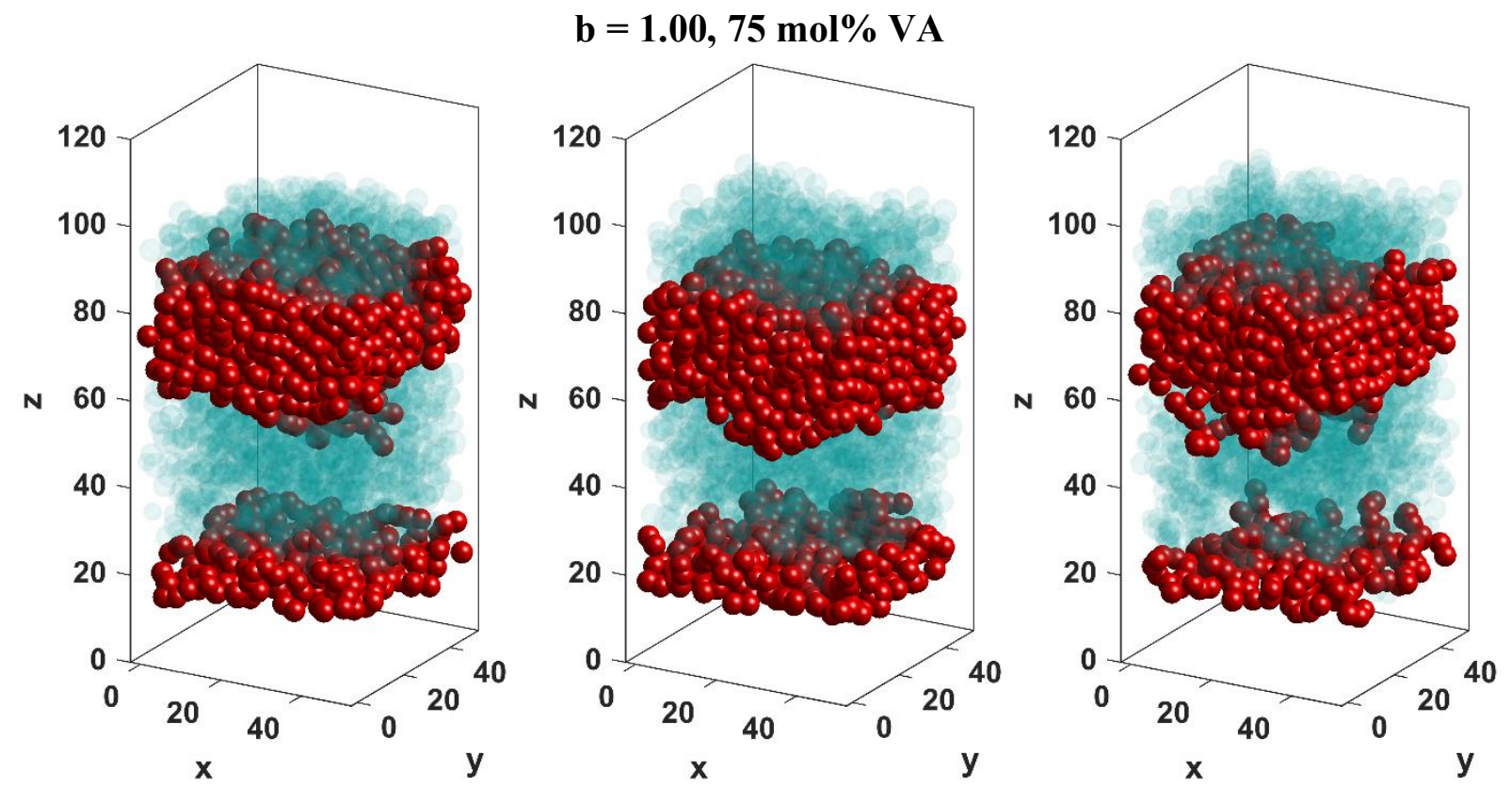

Figure S31. Morphology rendering for three independent sequence distributions with blockiness $\mathrm{b}=1.00$ and $75 \mathrm{~mol} \% \mathrm{VA}$ content. 


\section{S5. Additional adhesion simulation results: block averages of PVB-silica interaction energy}

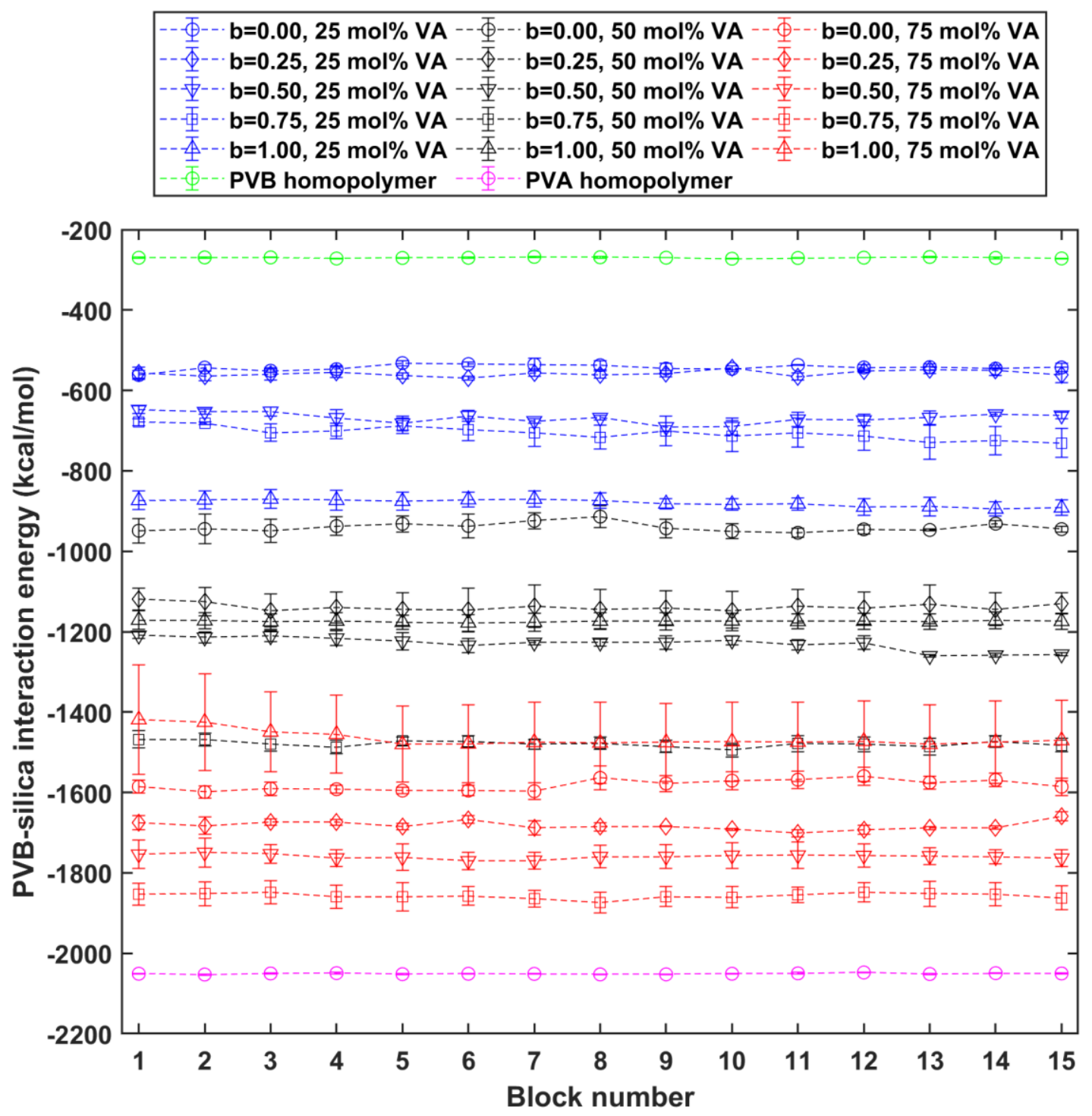

Figure S32. Adhesion energy in kcal per mol of beads for the PVB-silica interface for each blockiness and VA fraction, shown as averages taken over each 10ns block during equilibration at $400 \mathrm{~K}$. Error bars represent the standard error of the mean of the three independent trials. 
S6. Additional adhesion simulation results: volume fraction profiles, supplementary adhesion and loop, train, tail statistics.
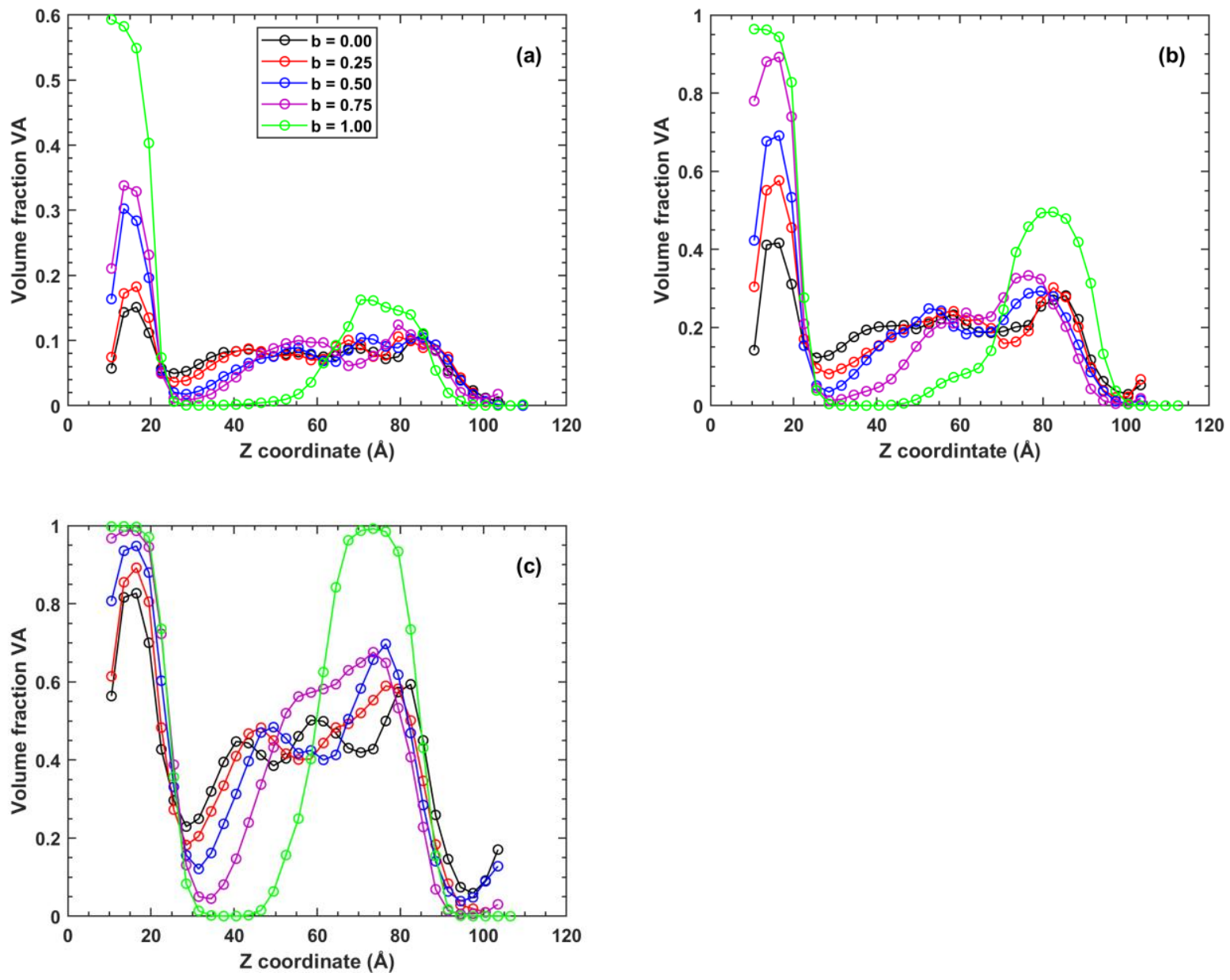

Figure S33. One-dimensional volume fraction profiles of VA monomers as a function of $\mathrm{z}$ coordinate (i.e., distance from the silica surface) and blockiness for (a) $25 \mathrm{~mol} \% \mathrm{VA}$, (b) $50 \mathrm{~mol} \%$ VA, and (c) $75 \mathrm{~mol} \% \mathrm{VA}$. Averages are taken over $100 \mathrm{~ns}$ at $400 \mathrm{~K}$, and the average of the three trials is shown. 

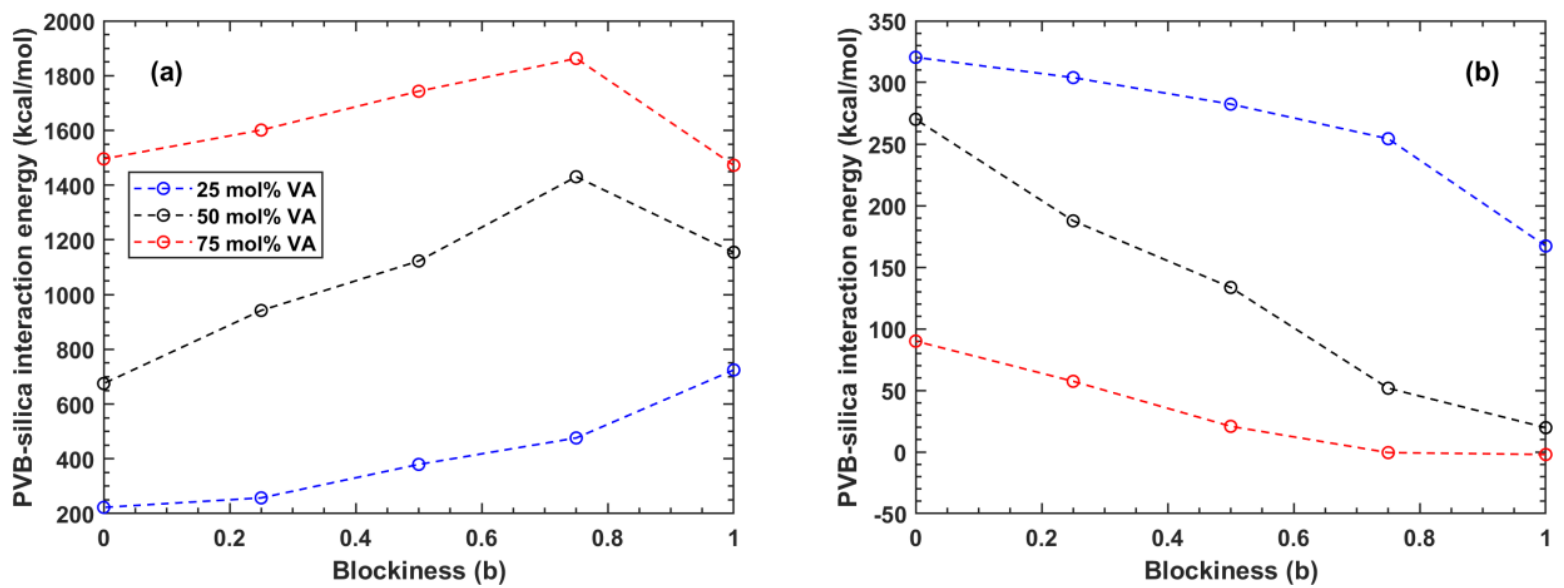

Figure S34. Contributions from the (a) VA and (b) VB monomers to the total adhesion energy shown in Fig. 8. 

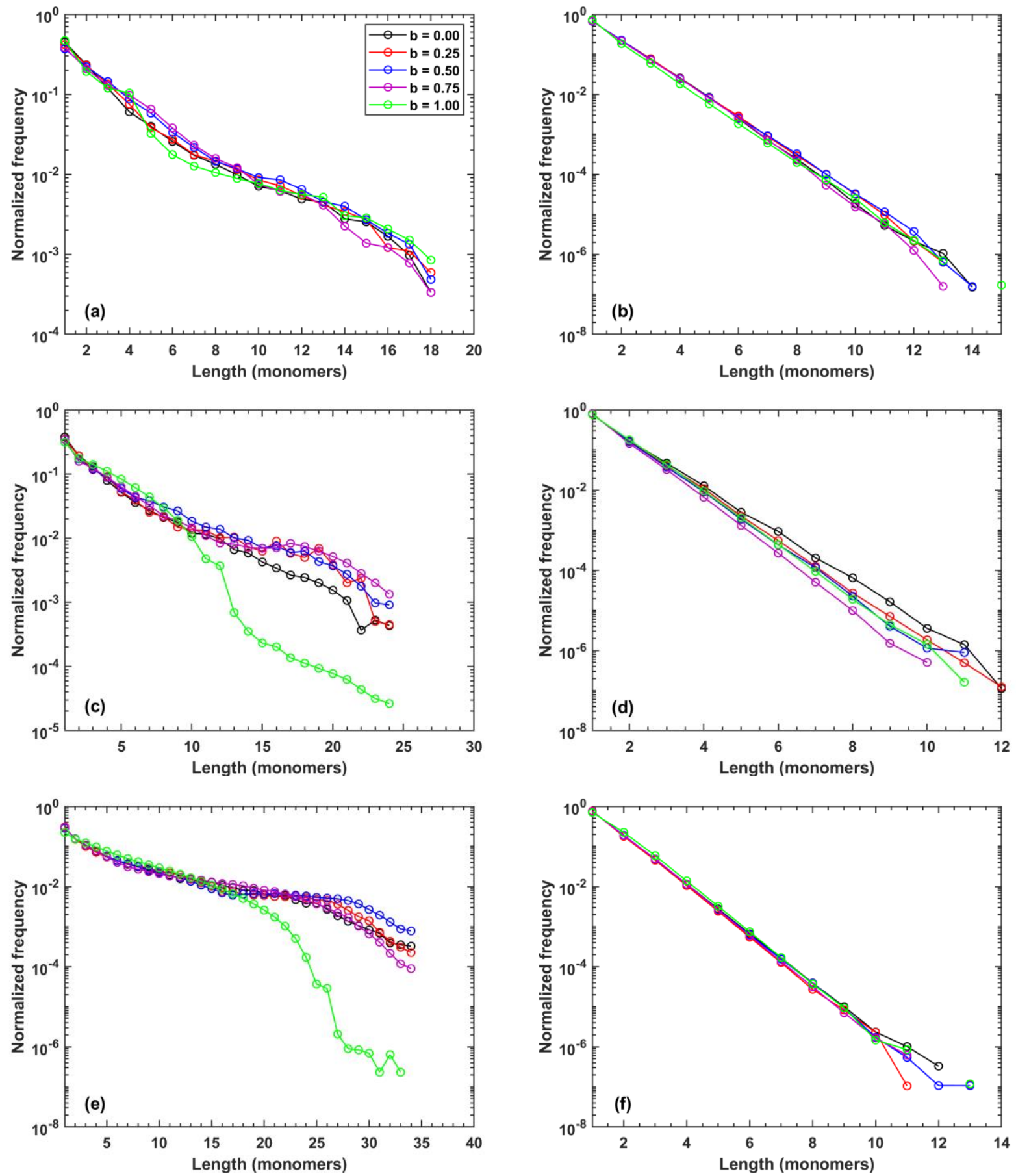

Figure S35. Loop length distributions for (a) $25 \mathrm{~mol} \% \mathrm{VA}$, (c) $50 \mathrm{~mol} \% \mathrm{VA}$, (e) $75 \mathrm{~mol} \% \mathrm{VA}$, and train length distributions for (b) $25 \mathrm{~mol} \% \mathrm{VA}$, (d) $50 \mathrm{~mol} \% \mathrm{VA}$, (f) $75 \mathrm{~mol} \% \mathrm{VA}$ as a function of blockiness ( $100 \mathrm{~ns}$ average at $400 \mathrm{~K}$ over three trials). The length distributions of tails, of which there are far fewer than trains and loops, are not sufficiently sampled to be of use. 

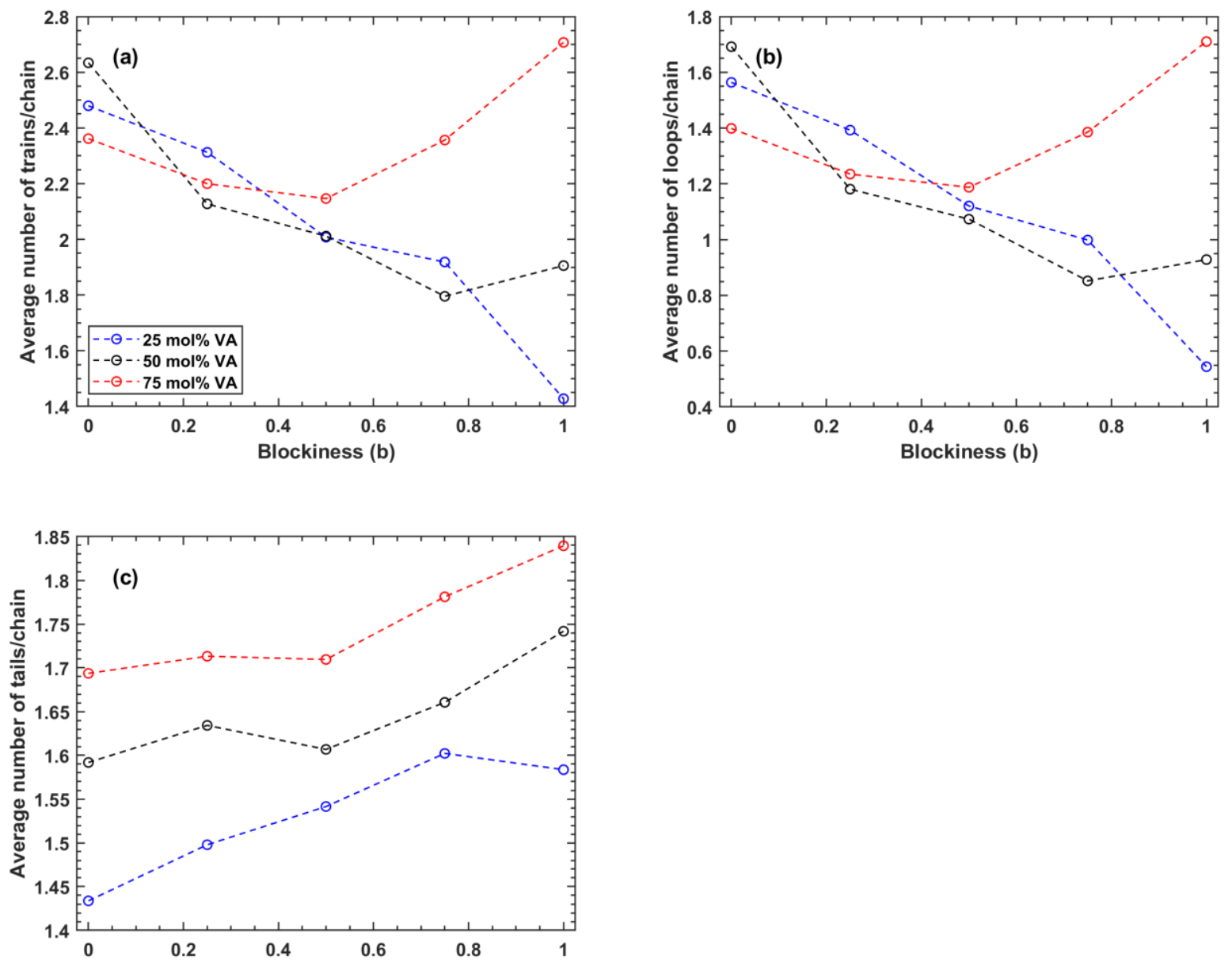

Figure S36. Average numbers of (a) trains, (b) loops, and (c) tails per adsorbed chain as a function of blockiness (100 ns average at $400 \mathrm{~K}$ over three trials). 


\section{REFERENCES}

(1) Plimpton, S. Fast Parallel Algorithms for Short - Range Molecular Dynamics. J. Comput. Phys. 1995, 117 (1), 1-19.

(2) Jorgensen, W. L.; Maxwell, D. S.; Tirado-Rives, J. Development and Testing of the OPLS All-Atom Force Field on Conformational Energetics and Properties of Organic Liquids. $J$. Am. Chem. Soc. 1996, 118 (15), 11225-11236.

(3) Lorenz, C. D.; Webb, E. B.; Stevens, M. J.; Chandross, M.; Grest, G. S. Frictional Dynamics of Perfluorinated Self-Assembled Monolayers on Amorphous SiO2. Tribol. Lett. 2005, 19 (2), 93-99.

(4) Hockney, R. W.; Eastwood, J. W. Computer Simulation Using Particles; CRC Press: New York, NY, 1988.

(5) Akima, H. A New Method of Interpolation and Smooth Curve Fitting Based on Local Procedures. J. ACM 1970, 17 (4), 589-602.

(6) MATLAB Release 2018a. The MathWorks, Inc.: Natick, Massachusetts. 